\title{
Transplantation of bacteriophages from ulcerative colitis patients shifts the gut bacteriome and exacerbates severity of DSS-colitis
}

Anshul Sinha*1, Yue Li*1,2, Mohammadali Khan Mirzaei ${ }^{1,3}$, Michael Shamash ${ }^{1}$, Rana

Samadfam $^{4}$, Irah L. King ${ }^{1,5+}$, Corinne F. Maurice ${ }^{1,5,6+}$

${ }^{1}$ Department of Microbiology \& Immunology, McGill University, Montreal, Quebec, Canada

${ }^{2}$ Department of Endocrinology and Metabolism, Guangdong Provincial Key Laboratory of Diabetology, The Third Affiliated Hospital of Sun Yat-Sen University, Guangzhou, Guangdong, 15 510630, China

${ }^{3}$ Institute of Virology, Helmholtz Center Munich and Technical University of Munich,

${ }^{6}$ Lead contact

*Authors contributed equally

+Correspondence: corinne.maurice@mcgill.ca,irah.king@mcgill.ca

\section{Keywords}

Bacteriophages, inflammatory bowel disease, DSS-colitis, ulcerative colitis, microbiota, intestine 


\section{ABSTRACT}

48 Inflammatory bowel diseases (IBDs) including Crohn's disease (CD) and ulcerative colitis (UC)

49 are characterized by chronic and debilitating gut inflammation. Altered bacterial communities of

50 the intestine are strongly associated with IBD initiation and progression. The gut virome, which is

51 primarily composed of bacterial viruses (bacteriophages, phages) is thought to be an important

52 factor regulating and shaping microbial communities in the gut. While alterations in the gut virome

53 have been observed in IBD patients, the contribution of these viruses to alterations in the bacterial

54 community and heightened inflammatory responses associated with IBD patients remains largely

55 unknown. Here, we performed in vivo microbial cross-infection experiments to follow the effects

56 of fecal virus-like particles (VLPs) isolated from UC patients and healthy controls on bacterial

57 diversity and severity of experimental colitis in human microbiota-associated (HMA) mice.

58 Shotgun metagenomics confirmed that several phages were transferred to HMA mice, resulting in

59 treatment-specific alterations in the gut virome. VLPs from healthy and UC patients also shifted

60 gut bacterial diversity of these mice, an effect that was amplified during experimental colitis. VLPs

61 isolated from UC patients specifically altered the relative abundance of several bacterial taxa

62 previously implicated in IBD progression. Additionally, UC VLP administration heightened colitis

63 severity in HMA mice, as indicated by shortened colon length and increased pro-inflammatory

64 cytokine production. Importantly, this effect was dependent on intact VLPs. Our findings build

65 on recent literature indicating that phages are dynamic regulators of bacterial communities in the

66 gut and implicate the intestinal virome in modulating intestinal inflammation and disease.

67

68

69 


\section{BACKGROUND}

71 The human gut microbiota is a complex community of microorganisms including bacteria, viruses,

72 archaea, and various eukarya, all of which provide protection against pathogens and maintain

73 metabolic and immunological homeostasis ${ }^{1,2}$. Intestinal bacterial communities are particularly

74 important in guiding the appropriate development of different immune cell types and regulating

75 the balance between pro- and anti-inflammatory responses in the gut ${ }^{2-6}$. For these reasons,

76 alterations in the gut bacteriome have been associated with various immunological disorders,

77 including inflammatory bowel diseases (IBDs) ${ }^{7}$. IBDs, comprised of Crohn's disease (CD) and

78 ulcerative colitis (UC), are chronic conditions in which regions of the gut are inflamed and

79 ulcerated, often leading to debilitating abdominal pain, rectal bleeding and diarrhea. In IBD

80 patients there is often a reduction in bacterial diversity, including a decrease in the proportion of

81 immunoregulatory short-chain fatty acid (SCFA)-producing Clostridia and an increase in tissue-

82 invasive Enterobacteriaceae ${ }^{7-10}$. Consistent with these clinical results, several of these bacterial

83 taxa have been shown to influence colitis severity in mouse models of intestinal inflammation ${ }^{11-}$

$84{ }^{15}$. In addition to these changes in the gut microbiota, population-based genetic studies have

85 revealed that several IBD risk-alleles are involved in host-microbe interactions ${ }^{16,17}$. Together,

86 these observations have led to the general assumption that IBD is the result of an inappropriate

87 intestinal immune response towards the gut microbiota in a genetically susceptible host. Despite

88 our understanding of genetics and changes to the gut microbiota in IBD, the precise factors that

89 drive bacterial alterations in IBD are not well understood.

90 Bacteriophages (phages), which are viruses that infect bacteria, are present at similar

91 abundances as their bacterial hosts in the human gut and have shown to be strong regulators of

92 bacterial communities in the mammalian gut ${ }^{18-22}$. Recent work has shown that in vivo 
93 administration of phages in mice can alter bacterial diversity ${ }^{21-24}$, disrupt bacterial interaction

94 networks ${ }^{19}$, and alter the concentration of bacterial-derived metabolites ${ }^{19}$. Importantly, there is

95 emerging data to support the idea that phages can alter disease outcomes by regulating the

96 composition and diversity of their bacterial hosts in the gut $21,24,25$.

97 Given the limitations of culturing gut bacteria and their associated phages, virome

98 characterization has primarily relied on metagenomic sequencing of fecal or gut mucosal samples.

99 However, due to the extensive diversity of phages and their low representation in databases, it has

100 been challenging to link phages to their bacterial hosts or gain taxonomic information from viral

101 sequence data alone ${ }^{26}$. Still, recent improvements in gut virome databases ${ }^{27,28}$ and bioinformatic

102 tools for detecting viruses ${ }^{29}$ have revealed some consistent characteristics of human gut viromes.

103 Specifically, phage communities from healthy adults are unique ${ }^{30}$, stable over time ${ }^{30,31}$, and

104 dominated by dsDNA Caudovirales phages and ssDNA Microviridae phages ${ }^{30,32-34}$. Compared to

105 other ecosystems, there also tends to be low virus-to-bacteria ratios (VBRs) ${ }^{20}$, a high proportion

106 of bacteria containing predicted prophages ${ }^{35}$, and a high prevalence of ubiquitous crAss-like

107 phages shown to infect Bacteroides in gut virome samples ${ }^{28,30,36}$.

Accumulating evidence from metagenomic sequencing of fecal and mucosal samples

109 indicates that the gut virome is altered in IBD patients, whether in CD or UC cohorts, and adults

110 or children ${ }^{37-40}$. Several of these reports have shown increases in the abundance and richness of

111 the order Caudovirales in IBD patients ${ }^{37-39}$. Some also report an increase in the relative abundance

112 of phages predicted to infect Firmicutes, which are typically reduced in IBD and contain several

113 species that induce anti-inflammatory immune responses ${ }^{37,41}$. Most recently, Clooney et. al 37

114 showed that, in addition to shifts in viral diversity, there was also increased relative abundance of

115 phages classified as temperate in UC and CD patients. These observations suggest that 
116 inflammatory events may initiate prophage induction in gut bacteria and a switch from the

117 lysogenic replication cycle to lytic replication ${ }^{37}$. Similar observations in mouse models of colitis

118 further support a link between intestinal inflammation and alterations of the gut virome ${ }^{42}$

119 These data collectively support a relationship between IBD and intestinal phage

120 communities. However, whether phage alterations impact gut bacterial communities, intestinal

121 immune responses, and/or disease progression is unknown. Here, we investigated the effects of

122 administering fecal virus-like particles (VLPs) from UC patients (UC VLPs) and non-IBD controls

123 (healthy VLPs) to human microbiota-associated (HMA) mice, and their subsequent impact on the

124 gut microbiome and dextran sodium sulfate (DSS)-induced colitis.

\section{RESULTS}

\section{Experimental model and composition of pooled viral and bacterial stocks}

128 In order to determine the effects of healthy and UC VLPs on bacterial and DSS-colitis severity,

129 we performed in vivo "cross-infection" experiments in HMA mice. Germ-free (GF) mice were

130 first colonized with pooled bacterial communities from 3 healthy volunteers or 3 UC patients

131 (healthy-HMA mice, UC-HMA mice). Following bacterial colonization, mice were given single

132 or multiple doses of VLPs, followed by 2\% DSS (see experimental schematics in Fig. 1). The

133 use of $2 \%$ DSS to induce colitis allowed for the temporal control of mild inflammation, thus

134 enabling us to study VLP-mediated effects on bacterial communities both independent of, and in

135 the presence of, intestinal inflammation. In addition, as DSS-induced inflammation is largely

136 restricted to the colon, our model mimics pathology similar to that observed in UC patients ${ }^{43}$.

To first characterize the virome of the pooled healthy and UC VLP stocks given to HMA

138 mice, we performed shotgun sequencing on VLP fractions from fecal samples. Quality-filtered 
139 reads from each sample were assembled into scaffolds, and viral scaffolds were detected and

140 annotated (see methods, Supplementary Fig. S1A). In total, 679 and 974 viral scaffolds were found

141 in the pooled healthy and UC VLP stocks, respectively (Fig. 2A, Supplementary Table. S1). In

142 agreement with the reported high inter-individuality of virome samples and differences in virome

143 composition between disease states ${ }^{30,37}$, only 37 scaffolds were shared between these stock

144 samples (Fig. 2A). We also used vConTACT2 to form viral clusters (VCs) based on shared protein-

145 coding genetic content in order to account for the high-inter individuality of these viromes ${ }^{44}$.

146 Using this approach, 478 and 641 VCs (including singletons) were found in the healthy VLP and

147 UC VLP stocks, respectively (Fig. 2A, Supplementary Table. S1). Of these VCs, only 89 were

148 shared between the 2 pooled stocks (Fig. 2A), suggesting that a substantial portion of these viral

149 stocks remained unique at this high taxonomic level. Using a vote-based approach to assign viral

150 taxonomy to the VLP scaffolds ${ }^{30}$, we also observed differences in the viral families present in each

151 stock (Fig. 2B). Using relative abundance, the healthy stock virome was predominately composed

152 of dsDNA phages belonging to the order Caudovirales and the families Myoviridae and

153 Siphoviridae and unclassified viral scaffolds (Fig. 2B). In contrast, the pooled UC stock virome

154 was dominated ( $76.36 \%$ relative abundance) by a single 6,339 bp scaffold belonging to the

155 (ssDNA) Microviridae family, along with phages belonging to the Siphoviridae and Podoviridae

156 families (Fig. 2B). These data are consistent with previous studies, showing that individual gut

157 viromes can be dominated by ssDNA Microviridae $30,32,34,37$. Based on the presence of integrase,

158 we were also able to classify viral scaffolds in our dataset as temperate ${ }^{29}$. The pooled UC stock

159 contained both higher absolute numbers of unique scaffolds identified as temperate and a higher

160 proportion of temperate scaffolds (Fig. 2C, Supplementary Table. S1), in line with previous

161 associations between temperate phages and IBD ${ }^{37}$. 
In addition to these differences in virome composition, we also used 16S rRNA gene

163 sequencing of the V4 region to determine the composition of the pooled healthy and UC bacterial

164 stocks used to colonize GF mice. Phylum-level analysis revealed decreased relative abundance of

165 Bacteroidetes (2.13\% UC, 36.32\% healthy), increased Actinobacteria (18.80\% UC, $4.63 \%$

166 healthy) and increased Proteobacteria ( $0.80 \%$ UC, $0.15 \%$ healthy) in the pooled UC stock (Fig.

167 2D, Supplementary Table. S1), consistent with previous observations of UC bacterial communities

$168 \quad 45-47$. We also compared genus-level differences between the bacterial stocks with differences in

169 genus-level bacterial host predictions of VLP scaffolds in our dataset using clustered regularly

170 interspaced short palindromic repeats (CRISPR) spacer homology ${ }^{48}$. In total, 186/679 (27.39\%)

171 of healthy VLP stock scaffolds were successfully assigned CRISPR spacer-based genus

172 predictions and 303/974 (31.11\%) of UC VLP stock scaffolds were assigned genus-level

173 predictions (Supplementary Table. S1). Some genus-level differences in relative abundance in the

174 pooled bacterial stock were consistent with differences in genus-level bacterial host predictions of

175 VLP scaffolds, indicative of concordance between the viral and bacterial fractions of these stocks.

176 For instance, increased relative abundance of Bifidobacterium in the UC bacterial stocks $(9.87 \%$

177 UC, $1.38 \%$ healthy) was consistent with a higher percentage of VLP scaffolds predicted to infect

178 Bifidobacterium (11.88\% UC, 3.23\% healthy) (Fig. 2E, Supplementary Table. S1). Additionally,

179 Prevotella was present at high relative abundances $(21.10 \%)$ in the healthy bacterial stock and was

180 not detected in the UC bacterial stock (Supplementary Table. S1). This disparity in Prevotella

181 abundance was reflected in a high proportion of Prevotella-infecting VLPs in the healthy stock

$182(17.74 \%)$ and zero scaffolds in the UC VLP stock predicted to infect Prevotella (Fig. 2E,

183 Supplementary Table. S1). Interestingly, Bacteroides-infecting VLPs made up $49.50 \%$ of

184 scaffolds with predicted CRISPR spacer hosts (Fig. 2E, Supplementary Table. S1), despite low 
185 Bacteroides relative abundance (1.79\%) in the UC bacterial inoculum (Supplementary Table. S1),

186 which could be reflective of an expansion of phages targeting and depleting Bacteroides in these

187 UC patients. Together, our data highlight UC-specific alterations in both the viral and bacterial

188 fractions of the pooled fecal samples used for VLP cross-infection experiments in HMA mice.

UC bacterial communities enhance DSS-colitis severity in comparison to bacterial

192 To first determine the effects of single healthy and UC VLPs doses on bacterial diversity and DSS-

193 colitis severity, we colonized GF mice with the pooled healthy and UC bacterial communities

194 described above (Fig. 2D). After 21 days of bacterial colonization, mice were given a single dose

195 of either healthy or UC VLPs, followed by 2\% DSS on day 30 (Fig. 1A). After a single dose of

196 VLPs, we did not observe differences in bacterial beta-diversity by weighted UniFrac distance

197 between mice given healthy or UC VLPs in either healthy or UC-HMA mice (Supplementary Fig.

198 S2A, Supplementary Table. S2). Using ANCOM, a statistical framework that accounts for the

199 underlying structure of microbial communities ${ }^{49}$, we were also unable to identify any species that

200 were differentially abundant between mice given healthy and UC VLPs in healthy or UC-HMA

201 mice during the VLP gavage period. Similarly, there were no significant differences in DSS-colitis

202 severity in HMA mice given healthy or UC VLPs (Supplementary Fig. S3), suggesting that single

203 doses of VLPs did not alter bacterial community composition or regulate intestinal inflammation.

204 However, regardless of whether healthy or UC VLPs were administered, UC-HMA mice

205 had increased colitis severity compared to healthy-HMA mice as determined by innate immune

206 cellular infiltration, inflammatory cytokine secretion in colonic explants, and tissue histology (Fig.

207 3A-F). Overall, these data are consistent with previous studies ${ }^{50,51}$ indicating that gut bacteria from 
208 UC patients predisposes HMA mice to an enhanced form of colitis. In order to determine the

209 differences between the gut bacterial communities in mice humanized with microbial communities

210 from UC patients or healthy volunteers, we performed 16S rRNA gene sequencing on mouse fecal

211 pellets. Principal coordinate analysis (PCoA) on weighted UniFrac distances including all time

212 points revealed significant differences in bacterial beta-diversity between healthy and UC-HMA

213 mice (Fig. 3G, PERMANOVA p= 0.001). We next tested for treatment-specific differences in

214 bacterial species previously associated with human IBD or experimental colitis severity. Using

215 ANCOM, we identified 61/189 species that were differentially abundant between mice given

216 bacterial communities from healthy volunteers and UC patients. HMA mice humanized with UC

217 bacteria showed reduced proportions of Akkermansia sp. across all time points (Fig. 3H), a

218 bacterial genus typically reduced in IBD patients and shown to ameliorate DSS-colitis ${ }^{12,52}$. It is

219 also well established that bacteria from the Enterobacteriaceae family increase in abundance in

220 IBD and exacerbate experimental colitis severity ${ }^{13,14}$. Accordingly, we found an expansion of

221 Escherichia-Shigella sp. during DSS-colitis, only in mice humanized with UC bacteria (Fig. 3I).

222 Importantly, in the bacterial stocks used to gavage the HMA mice, there were similar increases in

223 Escherichia-Shigella sp. (0.45\% UC, 0.069\% healthy) and decreases in Akkermansia sp. (0 \% UC,

$2242.14 \%$ healthy) (Supplementary Table. S1) in the UC samples compared to healthy volunteers.

225 Together, these colonization-specific differences in bacterial taxa may explain the exacerbation of

226 DSS-colitis in UC-HMA mice.

227

228

229 
232 We next wanted to determine whether multiple VLP doses from healthy volunteers could alter the

233 gut bacteriome and prevent the exacerbation of DSS-colitis. As we did not observe noticeable

234 changes in bacterial community composition (Supplementary Fig. S2A, Supplementary Table. S2)

235 or DSS-colitis severity (Supplementary Fig. S3) following a single dose of healthy or UC VLPs,

236 we explored whether multiple doses of healthy or UC VLPs would alter the gut microbiota of UC-

237 HMA mice (Fig. 1B-C). One common approach in phage therapy to increase treatment efficacy is

238 to add multiples doses instead of one single dose to sustain high phage densities ${ }^{53}$. Thus, for the

239 remaining experiments, we proceeded to give HMA mice multiple repeated doses of VLPs.

240 In the first experiment (Fig. 1B), UC-HMA mice were given four doses of healthy VLPs,

241 UC VLPs, or PBS over the course of 9-10 days. Following the VLP gavage period, mice were

242 given $2 \%$ DSS followed by a washout period. This first experiment was performed independently

243 twice (two trials). In a second experiment (Fig. 1C), we tested the impact of phage viability as well

244 as bacteria-independent effects of multiple doses of VLPs on intestinal inflammation using UC-

245 HMA mice administered intact or heat-killed UC VLPs and GF mice given UC VLPs alone (Fig.

246 1C). We first determined whether repeated dosing of VLPs would increase viral abundance and

247 virus-to-bacteria ratio (VBR) in HMA mice. Low levels of VLPs $\left(8.12 \times 10^{8}\right.$ virus $\left.\mathrm{mL}^{-1}\right)$ could be

248 detected in HMA mice during the bacterial colonization period (Fig. 4A) before the addition of

249 stock VLPs, likely a result of prophage induction of newly colonized bacteria and/or VLPs that

250 we were unable to remove during separation of the bacterial and VLP fractions in the stocks. Still,

251 these levels of VLPs during the bacterial colonization period were lower compared to after VLP

252 gavage (3.93-fold increase in UC VLP treated mice and 3.91-fold increase in healthy VLP treated 
253 mice) (Fig. 4A). Additionally, regardless of the source (healthy or UC samples), repeating VLP

254 dosing resulted in a significant increase in viral abundance and virus-to-bacteria ratio (VBR)

255 during the VLP gavage period relative to PBS (Fig. 4A-D, Supplementary Fig. 4A-D) or heat-

256 killed UC VLP controls (Supplementary Fig. 4E-H). Despite this, there was no associated decrease

257 in total bacterial abundance compared to PBS (Fig. 4B, Supplementary Fig. S4B) or to heat-killed

258 controls (Supplementary Fig. S4F), which suggests that bacteria killed by phage-mediated lysis

259 may be rapidly replaced by genetically or phenotypically resistant bacterial taxa ${ }^{19,54}$. Notably,

260 there was no detectable increase in viral abundance in GF mice given UC VLPs alone, suggesting

261 that phage replication required bacterial hosts (Supplementary Fig. S4E).

Since some ecological models predict that more metabolically active bacteria are less

263 susceptible to lytic phage infection ${ }^{55}$, we next determined if healthy and UC VLPs could alter the

264 proportion of active bacterial cells. Using SYBRGreen I staining and flow cytometry, we

265 determined the proportion of active fecal bacteria in HMA mice, as described previously ${ }^{56}$

266 (Supplementary Fig. S5A). Compared to PBS and heat-killed UC VLP controls, healthy and UC

267 VLPs did not shift the proportion of active bacteria (Supplementary Fig. S5C, S5E, S5G). We also

268 used Propidium Iodide (PI) staining to determine the proportion of damaged bacterial cells in

269 HMA mice (Supplementary Fig. S5B). Healthy and UC VLPs also did not shift the proportion of

270 damaged cells compared to heat-killed or PBS controls, with the exception of a single time-point,

271 where UC VLPs increased the proportion of damaged bacteria (Supplementary Fig. S5D, S5F,

272 S5H). Together, these data suggest that donor VLPs can interact with HMA mice but are not able

273 to shift the proportions of active or damaged gut bacterial cells to a detectable level. 
277 Shotgun sequencing was also performed on the VLP fraction of mouse fecal pellets to follow 278 changes in virome composition of HMA mice following VLP gavage. To assess the effectiveness

279 of transfer of VLPs from the pooled stocks to the HMA mice, we determined the proportion of 280 scaffolds found in the VLP stocks that were only found in HMA mice post-VLP gavage. In total, $28182 / 679(12.06 \%)$ of healthy VLP stock scaffolds were also found in HMA mice post healthy VLP 282 gavage (Supplementary Table. S3). In contrast, a higher number and proportion of UC VLP stock 283 scaffolds, reaching 210/974 (21.56\%), were found in HMA mice post UC VLP gavage, possibly 284 reflective of autologous transfer of UC VLPs to UC-HMA mice (Supplementary Table. S3). To 285 further assess the effectiveness of VLP transfer, we performed non-metric multidimensional 286 scaling (NMDS) on Jaccard distance of VCs and we measured the Jaccard distance of VCs between 287 each stock and HMA samples before and after transfer to HMA mice. Compared to the viromes of 288 mice before VLP gavage, there was a significant reduction in Jaccard distance between the viromes 289 of pooled healthy stock and the UC-HMA mice given healthy VLPs, which suggests that phages 290 present in the healthy stock are persisting in these mice after gavage (Fig. 5A, left). The same 291 comparison was made using UC VLPs without significant differences observed (Fig. 5A, right).

292 In line with these data, after VLP gavage, there was a significant increase in the richness of viral 293 scaffolds and VCs in UC-HMA mice given healthy VLPs and a non-significant increase in richness 294 of viral scaffolds in UC-HMA mice given UC VLPs compared to PBS controls (Supplementary 295 Fig. S1B).

296 We next performed NMDS on Bray-Curtis dissimilarity of viral scaffolds and VCs to 297 follow differences in viral beta-diversity over time in UC-HMA mice. As expected, there were no 
298 significant differences in Bray-Curtis dissimilarity during the baseline bacterial colonization 299 period (Fig. 5B, Supplementary Fig. S1C). In all cases, however, significant differences in viral 300 scaffold diversity were observed in all groups given VLPs compared to PBS controls, further 301 indicating successful transfer and replication of donor VLPs (Fig. 5B). Similar trends were 302 observed with VCs, however, there were no significant differences observed between healthy 303 VLP-treated mice and PBS controls during the VLP gavage period (Supplementary Fig. S1C, $304 \mathrm{p}=0.054)$. Notably, significant differences in viral diversity between all groups were maintained 305 during the DSS washout period (Fig. 5B, Supplementary Fig. S1C). To further assess differences between VLP-treated mice and PBS controls, we performed differential abundance analysis of 307 viral scaffolds using DESeq2 ${ }^{57}$. During the VLP gavage period prior to DSS administration, there 308 were 196 differentially abundant viral scaffolds between UC VLP-treated mice controls and PBS 309 controls (173 over-abundant, 23 under-abundant) and 272 differentially abundant scaffolds 310 between healthy VLP-treated mice and PBS controls (268 over-abundant, 4 under-abundant) (Fig.

311 5C, Supplementary Table. S4). During the DSS washout period, there were 303 differentially 312 abundant scaffolds between UC VLP-treated mice (209 over-abundant, 94 under-abundant) and 313 PBS controls and 232 differentially abundant scaffolds between healthy VLP-treated mice and 314 PBS controls (168 over-abundant, 64 under-abundant) (Supplementary Table. S4). Of these 315 significantly upregulated scaffolds, 46/173 (VLP gavage period) and 60/209 (DSS washout period) 316 in UC VLP-treated mice and 23/268 (VLP gavage period) and 34/168 (DSS washout period) in 317 healthy VLP-treated mice were also found in their respective pooled inoculums. Of the most over318 abundant scaffolds with CRISPR spacer-predicted hosts, mice given healthy VLPs were enriched 319 with Bacteroides-infecting phages during both the VLP gavage and DSS washout periods 320 (Supplementary Table. S4). In contrast, UC VLP-treated mice were enriched with phages predicted 
321 to infect Hungatella, Barnsiella, Erysipelatoclostridium, and Bacteroides during the VLP gavage

322 period and were enriched with Bacteroides, Faecalibacterium and Eubacterium phages during the

323 DSS washout period (Supplementary Table. S4). Together, these data indicate that VLPs were

324 transferred from pooled stocks to HMA mice, with distinct changes in viral beta-diversity and

325 potential phage-bacterial interactions following VLP gavage.

\section{VLPS derived from healthy individuals or UC patients have distinct effects on the UC gut}

bacterial communities in vivo

329 In order to identify whether multiple healthy and UC VLPs doses had distinct effects on bacterial 330 community composition, we performed $16 \mathrm{~S}$ rRNA gene sequencing on mouse fecal pellets before 331 and after VLP administration, as well as during the DSS/washout period. Using ANCOM, we 332 identified bacterial species differentially abundant between UC-HMA mice given multiple doses 333 of either UC VLPs, healthy VLPs, or PBS (summarized in Supplementary Tables. S5-8). To

334 account for variation due to isolator-specific differences during colonization, bacterial species that 335 were differentially abundant during the baseline bacterial colonization period were not included.

336 In addition, the remaining differentially abundant species were each ranked based on the likelihood

337 that treatment-specific differences were due to VLP treatment or isolator effect (1: likely due to

338 VLP treatment, 2: possibly due to VLP treatment, 3: likely due to isolator effect, see methods for 339 ranking criteria).

340 Across the two trials where UC-HMA mice were given multiple doses of healthy VLPs, 341 UC VLPs, or PBS, 11 differentially abundant species (4/90 species trial \#1; 7/85 species trial \#2) 342 were identified in the VLP gavage period before DSS administration, and 19 species (4/94 species 343 trial \#1; 15/85 species trial \#2) were identified in the DSS/washout period (Supplementary Tables. 344 S5-6). Between mice given multiple doses of UC VLPs (+/- DSS) or heat-killed UC VLPs, 3/49 
345 differentially abundant bacterial species were identified in the VLP gavage period and 6/47 species

346 were identified in the DSS/washout period (Supplementary Tables. S7-8). Some of these

347 differentially abundant bacterial species have been shown to influence experimental colitis

348 severity, or to be differentially abundant in CD or UC patients. For example, Eubacterium limosum

349 was significantly reduced in mice given UC VLPs (Fig. 6A, left), consistent with its ability in

350 mouse models to ameliorate DSS-colitis severity ${ }^{58}$. We also observed that Enterococcus sp., which

351 have been shown to play divergent roles in the context of DSS-colitis severity ${ }^{59,60}$, were increased

352 in relative abundance in HMA mice (Fig. 6A, middle). In addition, compared to heat-killed UC

353 VLPs, mice given intact UC VLPs showed a significant increase in the proportion of Escherichia-

354 Shigella sp. after VLP gavage (Fig. 6A, right). Given that Enterobacteriaceae are increased in IBD

355 patients and are thought to exacerbate dysregulated immune responses in IBD ${ }^{7,13,61}$, these data

356 suggest that UC VLPs may allow for the expansion of this species.

357 PCoA on weighted UniFrac distances revealed no significant differences in bacterial beta-

358 diversity of HMA mice during the bacterial colonization period and during the following 9-day

359 period where 4 doses of VLPs (healthy, UC, or PBS) were given to these mice (Fig. 6B). In

360 contrast, we observed significant differences in bacterial community composition between mice

361 given healthy VLPs, UC VLPs, or PBS following DSS administration, supporting the idea that

362 these phages have distinct effects on bacterial communities, which are amplified during DSS

363 colitis (Fig. 6B). Similar trends were observed in a second independent trial (Supplementary Fig.

364 S2B, Supplementary Table. S2). In line with these data, there were significant differences in

365 bacterial diversity between UC-HMA mice given UC VLPs (+DSS) compared to those given heat-

366 killed UC VLPs (+DSS) (Supplementary Fig. S2C, Supplementary Table. S2), suggesting that

367 intact viruses are necessary for driving changes in bacterial diversity. In contrast, we did not 
observe differences in weighted UniFrac distances during the DSS/washout period between UC-

369 HMA mice given UC VLPs in the absence of DSS, compared to heat-killed UC VLPs

370 (Supplementary Fig. S2C, Supplementary Table. S2). These data further support the idea that

371 phage-mediated changes in gut bacterial communities are initially modest, but then amplified

372 during intestinal inflammation. In order to investigate whether these greater changes in bacterial

373 diversity following DSS administration were in part due to prophage induction as a result of

374 intestinal inflammation, we also determined the proportion of VLP scaffolds identified as

375 temperate following DSS. Within each treatment group, there was a modest increase in the relative

376 abundance of VLP scaffolds identified as temperate following DSS (Supplementary Fig. S1D).

377 We also identified significantly over-abundant scaffolds after DSS-administration within each

378 treatment group that were classified as temperate. Despite the modest increase in temperate VLP

379 relative abundance following DSS, only 1/16 (UC VLP treatment), 3/8 (healthy VLP treatment)

380 and $8 / 30$ (PBS control) of the over-abundant scaffolds were identified as temperate

381 (Supplementary Table. S4), suggesting that any DSS-mediated effects on prophage induction are

382 likely modest. To determine whether there was experimental evidence for prophage induction due

383 to direct interactions between DSS and UC bacterial communities, we performed an in vitro

384 prophage induction assay ${ }^{62-64}$. In UC bacterial cultures grown anaerobically in the presence of

385 DSS, we did not detect an increase in VLPs in the bacterial supernatant (Supplementary Fig. S1E),

386 suggesting that any DSS-mediated prophage induction occurring in HMA mice is likely not due

387 to direct DSS toxicity to bacterial cells and is likely through DSS-mediated induction of

388 downstream immune responses.

389 Together, these data indicate that healthy and UC VLPs drive distinct changes in the

390 relative abundance of bacterial species in UC-HMA mice, some of which have been shown to 
391 influence experimental colitis severity and IBD disease progression. In addition, while several

392 bacterial species were found to be differentially abundant during the VLP gavage period,

393 differences in bacterial diversity between VLP-treated mice are greatest during DSS-colitis,

394 suggesting that intestinal inflammation may provide a more conducive environment for phage-

395 mediated changes in these gut bacterial communities.

\section{UC VLPS increase colitis severity in HMA mice}

398 Given that the healthy and UC VLPs are distinct in their composition and contribute differently to

399 bacterial community composition, we next tested if they had distinct effects on colitis outcomes.

400 We first determined whether there were differences in colitis severity between UC-HMA mice

401 given healthy VLPs, UC VLPs, or PBS. Upon DSS administration, a slight decrease of body

402 weight could be seen only in mice administered UC VLPs (Fig. 7A). Importantly, at day 10 post-

403 DSS challenge, only the weight of UC-HMA mice given UC VLPs was declining (Fig. 7A). HMA

404 mice given UC VLPs also showed a significant shortening of colon length (Fig. 7B) and an

405 increase of pro-inflammatory cytokine production (Fig. 7C), indicating that UC VLPs can

406 exacerbate the severity of DSS colitis compared to healthy VLPs and the PBS control.

407 To further investigate the function of UC VLPs on the pathology of DSS-induced colitis,

408 we next assessed DSS-colitis severity between UC-HMA mice given (i) UC VLPs with DSS; (ii)

409 UC VLPs without DSS to determine if UC VLPs can cause spontaneous colitis; (iii) heat-killed

410 UC VLPs to determine if intact VLPs are necessary for increased colitis severity; and (iv) GF mice

411 given UC VLPs to determine if increased colitis severity was a result of direct UC VLP-immune

412 interactions (Fig. 7C).

413 Consistent with our previous results (Fig. 7C), UC-HMA mice given UC VLPs had a

414 significantly shortened colon and produced the most TNF- $\alpha$ and IL-1 $\beta$ from colon explants 
415 compared to the other UC-HMA groups (Fig. 7D-F). Notably, the heat-killed UC VLP group mice

416 showed significantly less severe colitis compared to the intact UC VLPs group, suggesting the

417 changes we observe are due to viral infection rather than the presence of phage components alone

418 (Fig. 7D-F). Finally, we found that GF mice given UC VLPs prior to DSS treatment exhibited the

419 greatest colon shortening, yet least inflammatory cytokine production (Fig. 7D-F). These results

420 are similar to our observations of increased colonic shortening in GF mice compared to HMA mice

421 (Supplementary Fig. S6) and suggest that, without bacterial hosts, phages cannot modulate colitis

422 outcomes.

\section{DISCUSSION}

425 Phages are known regulators of bacterial diversity and metabolism in several environments, 426 including the mammalian gut $18,65,66$. In IBD, metagenomic analyses have revealed that the virome 427 composition of CD and UC patients is altered in comparison to non-IBD controls ${ }^{37-40}$. However, 428 experimental work investigating the interactions between phages, intestinal bacterial communities, 429 and immune responses in the context of IBD is limited ${ }^{67}$. Given the importance of gut bacterial 430 communities in human health, phage-mediated changes of the microbiota are thought to have 431 important physiological consequences ${ }^{26}$. Here we show that VLPs isolated from healthy 432 volunteers and UC patients differentially modulate the composition of the gut microbiota, and that 433 administration of multiple doses of UC VLPs increases colitis severity in UC-HMA mice.

434 Four doses of UC VLPs and healthy VLPs significantly altered the fecal bacteriome of UC-

435 HMA mice compared to PBS and heat-killed UC VLP-treated controls. This was not seen when a 436 single dose of UC VLPs was used, supporting the idea that the regulatory effect of phages in the 437 mammalian gut is dose dependent ${ }^{23}$. The differences in the effects of multiple doses compared to 
438 single doses of VLPs on bacterial community composition and downstream disease outcomes have

439 important implications for targeted or whole community phage therapy approaches in the gut ${ }^{26}$.

$440 \quad$ Several bacterial species were found to be differentially abundant during the VLP gavage

441 period prior to DSS-colitis, suggesting that VLPs had distinct effects on the bacterial community

442 in the absence of induced inflammation. However, we only observed significant differences in

443 bacterial beta-diversity during the DSS/washout period, highlighting that these initial VLP-

444 mediated effects are amplified during the DSS-colitis/washout period. The disrupted colonic

445 mucus layer, potential changes in bacterial growth rate, and/or innate immune cell-derived

446 mediators in the inflamed gut could all provide a more conducive environment for increased phage-

447 host interactions ${ }^{55}$. Given these drastic changes in the gut environment during inflammation, it is

448 possible that this facilitates a switch from Piggyback-the-Winner dynamics, common in the

449 mammalian gut, to Kill-the-Winner dynamics ${ }^{55}$. Changes in microbial expression profiles in IBD

450 patients ${ }^{68}$ and in murine experimental colitis ${ }^{69}$ could also alter bacterial susceptibility to phage

451 infection.

452 The use of DSS-colitis as a model of inflammation is limited in that the immune responses

453 elicited and the exaggerated damage to the epithelium differ from clinical UC ${ }^{70}$. However, we

454 induced a mild colitis, as HMA mice given 2\% DSS experienced minimal weight loss and blood

455 in their stool. In addition, the reproducibility and temporal control offered by DSS were valuable

456 in our approach to study the effects of VLPs on bacterial communities in the context of broad

457 inflammatory progression and changes. Thus, our data provide the framework for future studies

458 investigating phage-host interactions during inflammation. Given the modest increase in the

459 relative abundance of temperate phages observed across treatment groups following DSS,

460 prophage induction resulting from DSS-induced inflammation may also contribute to these 
461 differences in bacterial diversity. Only a small fraction of differentially abundant scaffolds were

462 identified as temperate during the DSS washout period, yet we are likely underestimating this

463 number since all samples collected during DSS exposure could not be used for VLP shotgun

464 sequencing due to DSS-mediated inhibition of DNA polymerase required for multiple

465 displacement amplification (MDA), despite our attempts to purify DNA using spermidine ${ }^{71}$.

466 Interestingly, while we did observe differences in bacterial community diversity based on VLP

467 treatment, there were no differences in bacterial activity or damage in VLP treated mice,

468 suggesting that these changes were too modest to detect at a whole bacterial community level.

As previously observed, there were differences in virome composition between the healthy

470 and UC fecal VLP and bacterial stocks ${ }^{7,37-40}$. However, contrasting with previous metagenomic

471 analyses of IBD viromes, there was not a high relative abundance of dsDNA Caudovirales phages

472 in our pooled UC VLP stock. Instead, the UC virome was dominated by a single 6,339 bp scaffold

473 classified as Microviridae. Our limited sample size to prepare the pooled phage stocks, the known

474 high inter-individual differences in gut viromes ${ }^{30}$, differences in virome composition based on

475 viral enrichment and DNA extraction strategies ${ }^{28,72,73}$, and the known bias in the amplification of

476 ssDNA viruses using multiple-displacement amplification (MDA) ${ }^{74,75}$ are all possible causes that

477 could explain these differences between the virome composition in our dataset and previously

478 published viromes of IBD patients. Still, our observations of increased proportions of temperate

479 phages in the UC VLP stock, and alterations in Bacteroidetes, Actinobacteria, and Proteobacteria

480 in the UC bacterial stock are consistent with previously reported UC-specific alterations of the

481 microbiota ${ }^{37,45-47}$. While our strategy to pool the healthy and UC bacterial and VLP stocks comes

482 with the loss of inter-individual genomic information between samples within a given treatment

483 group, pooling stocks maintains intra-specific information while minimizing variation during 
484 transplantation, especially important given the stochasticity during microbiota acquisition ${ }^{76}$ and

485 low percentage of retained taxa following transplantation ${ }^{77}$. Importantly, we assessed the

486 composition of the pooled viral and bacterial stocks in addition to following changes in microbiota

487 of HMA mice over time, thus allowing us to assess the efficacy of bacterial and VLP engraftment ${ }^{78}$.

488 Additionally, despite the limitations of this approach, compared to previous studies that have

489 investigated VLP-mediated changes to bacterial diversity or disease severity in mice ${ }^{21,23,24}$, our use

490 of HMA mice and human-derived VLPs adds translatability of our findings to the microbiota

491 alterations observed in UC patients.

492 Importantly, we observed differences in viral beta-diversity post-VLP gavage and

493 identified scaffolds transferred from the pooled VLP stocks to HMA mice, suggesting that phages

494 within the healthy and UC stocks were able to successfully engraft in recipient mice. While VLP

495 shotgun sequencing could not be performed during DSS-colitis, differences in viral diversity

496 persisted into the DSS washout period, suggesting that viromes of mice given healthy and UC

497 VLPs were divergent before and after inflammation. Successful engraftment was also supported

498 by increased viral abundance after VLP gavage and a significant decrease in Jaccard distance of

499 VCs to the healthy stock virome following healthy VLP gavage. While the decrease in Jaccard

500 distance of VCs to the UC stock virome was not significant, these data may be explained by the

501 fact that phages present before VLP gavage in HMA mice colonized by UC bacteria may already

502 resemble the UC stock since they all come from the same initial UC patients. In addition, the post-

503 gavage viromes of healthy and UC VLP-treated were characterized by distinct phage-host

504 interactions, as predicted by CRISPR spacer matches. The treatment-specific differences in the

505 predicted bacterial hosts that these phages target are in support our observations that healthy and

506 UC VLPs have divergent effects on gut bacterial communities. The particular observation that 
507 healthy VLPs successfully engrafted in HMA mice colonized with UC bacterial communities is

508 consistent with previous observations of phage "cross-reactivity" between different individuals or

509 disease states and likely supports the idea that some phages in the gut have host-ranges at the

510 species rather than the strain level ${ }^{21,23,24,79,80}$. Notably, our functional annotation of viral scaffolds

511 revealed no obvious differences in the auxiliary metabolic genes present between treatment groups

512 (data not shown), suggesting shared potential functionality between viromes.

513 Regardless of whether HMA mice were given a single dose of healthy or UC VLPs, mice

514 colonized with fecal bacteria isolated from UC patients had increased colitis severity compared to

515 mice colonized with fecal bacteria from healthy donors. This finding is consistent with previous

516 reports showing exacerbation of DSS-induced and T cell-mediated colitis following administration

517 of gut microbiota from IBD patients to germ-free mice ${ }^{50,51}$. While these studies associated an

518 increase of IL-17 producing CD4+ T cells to disease severity, anti-viral immunity is more

519 commonly associated with type 1 immunity and interferon production. As such, an impact of the

520 virome on $\mathrm{T}$ cell immunity is likely indirect via changes to bacteria-derived products that promote

521 Th17 cell differentiation. Nevertheless, phages express pathogen-associated molecular patterns

522 that, if accessed by host immune cells, may directly alter tissue inflammation ${ }^{81}$. Although we

523 administered UC VLPs to GF mice in order to investigate this scenario, VLPs were cleared in the

524 absence of bacterial hosts, resulting in undetectable changes to the host immune response.

Two important limitations in using HMA mice to monitor disease outcomes are that (1)

526 only a fraction of human fecal bacteria can colonize GF mice ${ }^{77}$ and (2) there is a reported

527 colonization bias towards Bacteroidetes ${ }^{77}$. Despite these limitations, we identified differences in

528 the relative abundances of certain bacterial species in mice after colonization with healthy or UC

529 fecal bacteria, which could explain the differences in colitis severity between these two groups. 
530 This included Akkermansia sp., which are reduced in IBD patients and have been shown to be

531 protective in the context of DSS-colitis ${ }^{12}$, and Escherichia-Shigella sp., which are thought to

532 exacerbate the pro-inflammatory immune responses in IBD ${ }^{82}$. We speculate that the increased

533 colitis severity observed in UC-HMA mice given UC VLPs compared to mice given PBS, heat-

534 killed UC VLPs, or healthy VLPs may be due to alterations of the gut bacterial communities

535 resulting from phage predation. Some of the bacterial species reduced in relative abundance by

536 UC VLPs, such as E. limosum ${ }^{58}$ and Ruminoclostridium 5, are thought to be producers of short-

537 chain fatty acids (SCFAs), which are important in promoting epithelial barrier function,

538 antimicrobial peptide production, and induction of immunomodulatory Tregs ${ }^{3,9}$. Given that IBD

539 patients have reduced fecal SCFA concentrations ${ }^{83,84}$, these data support the idea that UC VLPs

540 may cause increased colitis severity by depleting these SCFA-producing bacteria. In support of

541 this idea, we found that the reduction of E. limosum during the DSS/washout period in UC VLP-

542 treated mice coincided with an enrichment of phages predicted to infect Eubacterium. However,

543 given the current limitations in bioinformatically matching phages to their hosts (we were only

544 able to assign predicted hosts to $\sim 30 \%$ of our viral scaffolds) and the diverse interbacterial

545 interactions in the gut, it is difficult to definitively attribute the phage predation of specific bacterial

546 taxa to an altered disease pathology. Notably, UC VLP administration also led to an increase in

547 the relative abundance of Escherichia-Shigella sp., possibly an indirect result of phage predation

548 and its subsequent modulation of the gut microbiota through interbacterial interactions, as recently

549 described by Hsu et al. ${ }^{19}$. Consistent with the notion that Proteobacteria can contribute to IBD

550 pathogenesis ${ }^{13}$, our data indicate that phage regulation of bacterial communities promotes the

551 expansion of immune-activating, potentially pathogenic bacteria. In agreement with our earlier

552 observations, Khan Mirzaei et al. ${ }^{80}$ showed that phages from stunted children promoted the in 
553 vitro expansion of Proteobacteria, which are important in contributing to the nutritional

554 deficiencies of this disease. The observation that VLPs isolated from the patients of two distinct

555 diseases allowed for the expansion of Proteobacteria across different ages and experimental

556 settings has important relevance for virome alterations in inflammatory diseases and warrants

557 further investigation. While it is possible that endotoxin remaining in the VLP preps also

558 contributed to the observed differences in colitis severity, mice given heat-killed UC VLPs

559 displayed reduced colitis severity compared to intact UC VLPs, suggesting that LPS contamination

560 does not explain these effects.

\section{CONCLUSIONS}

562 While several studies have outlined gut virome alterations in IBD patients, our observations

563 that fecal VLPs from UC patients exacerbate DSS-colitis severity suggest that these alterations

564 could be important for IBD pathogenesis and gut inflammation. This action may be either through

565 phage-mediated changes in the microbiota or by direct interactions with the intestinal immune

566 system. Overall, the power of experimental in-vivo cross infections performed here allowed us to

567 highlight a causal role for phages in modulating gut bacterial communities and disease outcome. 
FIGURES AND FIGURE LEGENDS

577
A
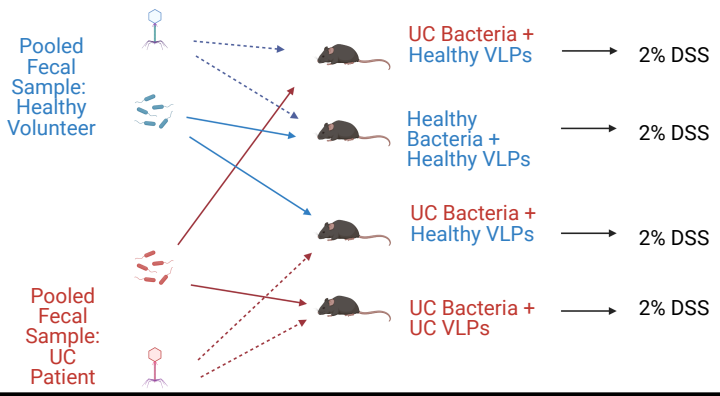

B

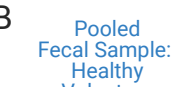
Volunteer
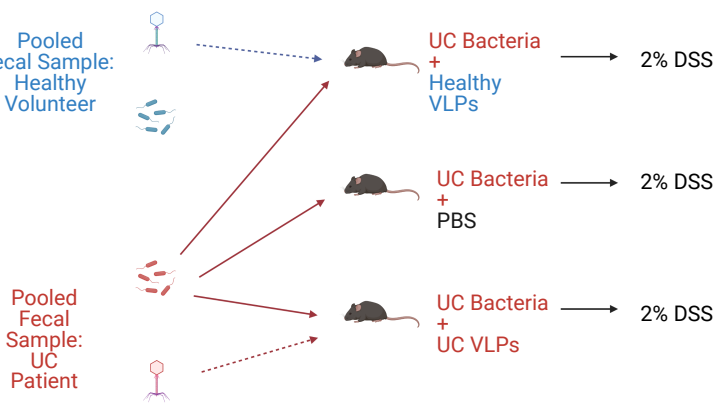

C

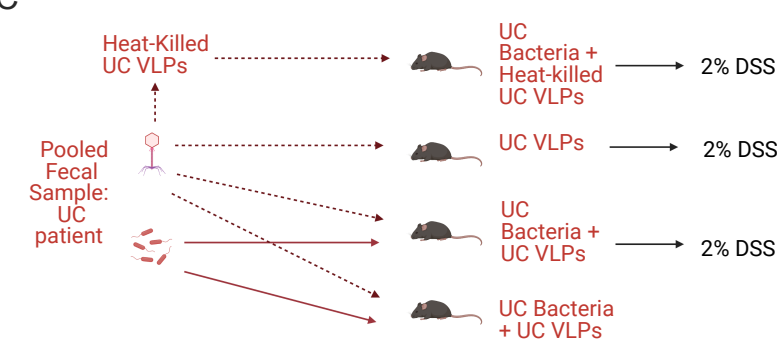

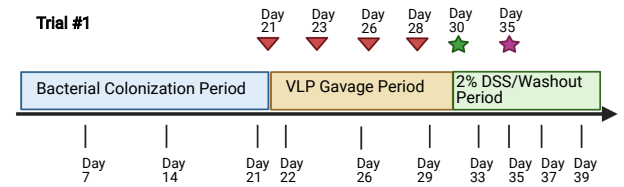
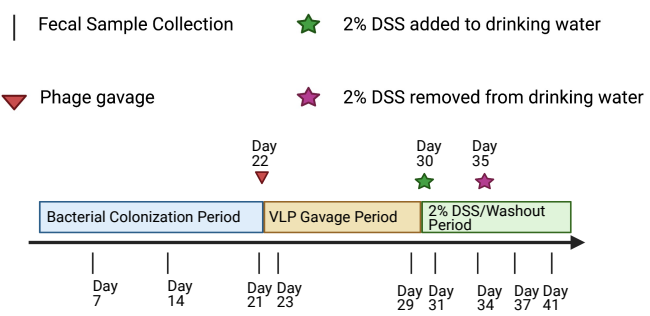

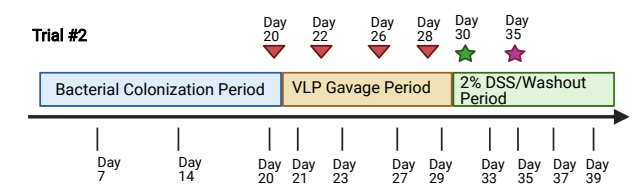

Figure 1. Schematic and timeline of experimental model. (A) HMA mice administered healthy or UC bacterial communities were given a single dose of healthy or UC VLPs, followed by $2 \%$ DSS. (B) UC-HMA mice were given four doses of healthy VLPs, UC VLPs or PBS, followed by 2\% DSS. (C) UC-HMA or GF mice were given four doses of UC VLPs or heat-killed UC VLPs. All groups were then given 2\% DSS, except one group of UC-HMA mice given UC VLPs. Each treatment group included 5 or 6 GF or HMA mice per experiment housed in three separate cages. In each experiment $200 \mu \mathrm{L}$ of bacterial and VLP communities were administered to mice by oral gavage at equal concentrations (1-3 x $10^{8}$ VLPs or bacterial cells $\left./ \mathrm{mL}\right)$. 
bioRxiv preprint doi: https://doi.org/10.1101/2021.09.10.459444; this version posted September 11, 2021. The copyright holder for this preprint (which was not certified by peer review) is the author/funder, who has granted bioRxiv a license to display the preprint in perpetuity. It is made available under aCC-BY-NC 4.0 International license.

A

$$
\text { Viral Scaffolds }
$$

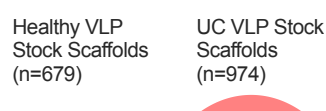

623

624

625

626

627

628

629

630

631

632

633

634

635

636

637

638

639

640

641

642

643

644

645

646

647

648

649

650
B

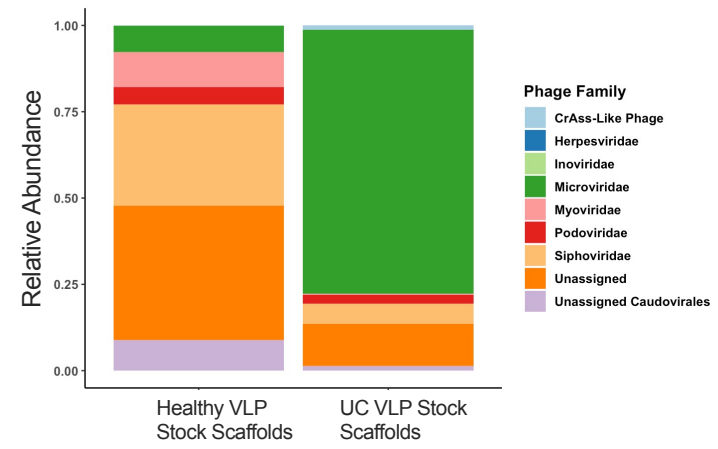

D

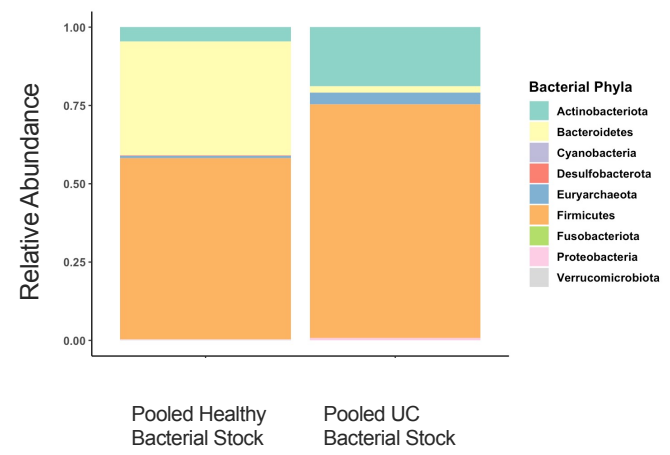

Viral Clusters (VCs)

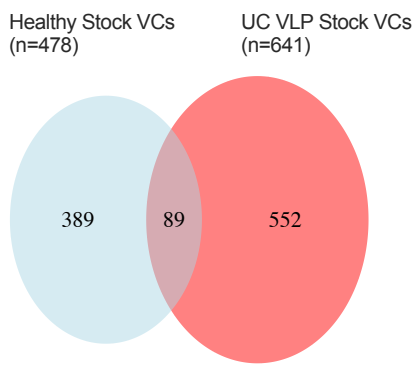

C

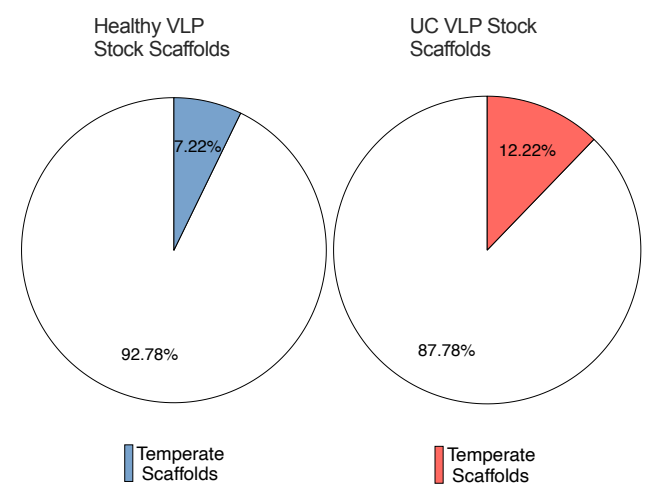

E

$\begin{array}{ll}\text { Healthy VLP } & \text { UC VLP Stock } \\ \text { Stock Scaffolds } & \text { Scaffolds }\end{array}$
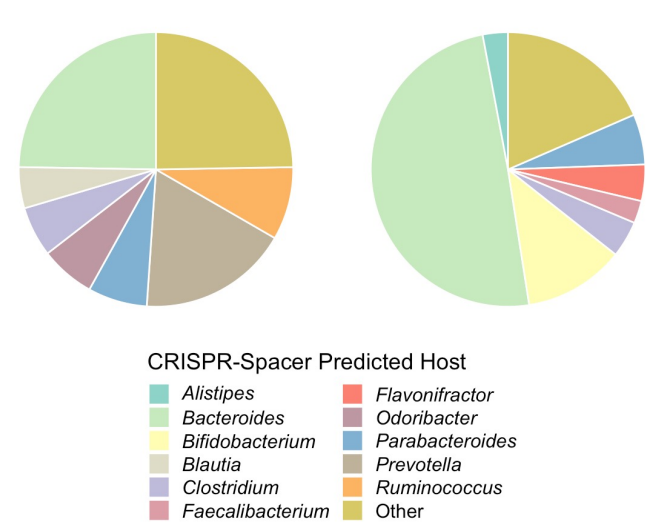

Figure 2. Composition of pooled healthy and UC VLP and bacterial stocks (A) Shared and unique viral scaffolds and VCs between pooled healthy and UC VLP stocks. (B) Relative abundance of viral families in each VLP stock. (C) Proportion of scaffolds in VLP stocks identified as temperate. (D) Relative abundance of bacterial phyla in each pooled bacterial stock. (E) Proportion of scaffolds based on CRISPR spacer predicted hosts. The top 7 most prevalent host predictions in each treatment group are displayed. VLP shotgun metagenomics was used for VLP stock analyses and 16S rRNA gene sequencing of the V4 region was used for bacterial stock analyses. Stock samples were pooled using equal weight of 3 fecal samples from healthy volunteers or UC patients. 

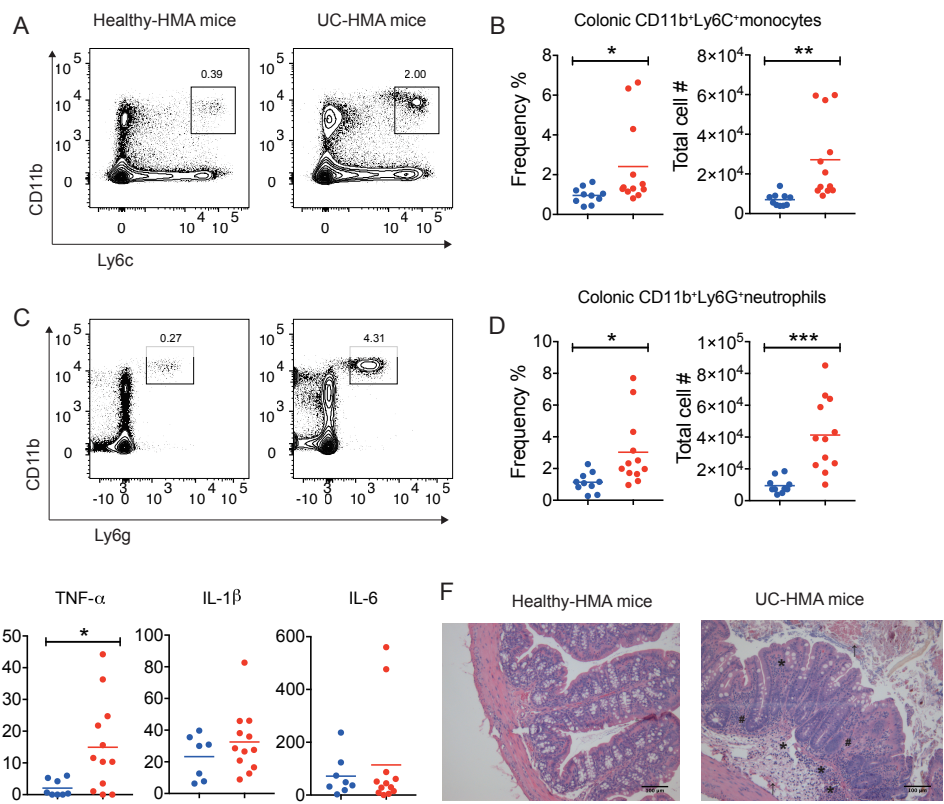

G

H

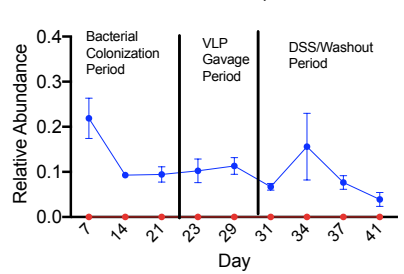

665

666

- Healthy-HMA mice

- UC-HMA mice

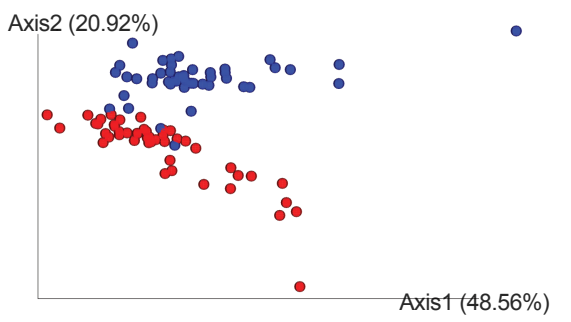

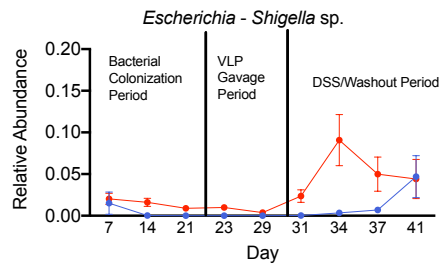

Figure 3. Mice colonized with UC patient-derived fecal bacteria exhibit increased inflammation during experimental colitis. (A) Representative contour plots and (B) mean frequency and absolute number of colonic inflammatory monocytes $\left(\mathrm{CD}^{11} \mathrm{~b}^{+} \mathrm{Ly} 6 \mathrm{C}^{+} \mathrm{Ly} 6 \mathrm{G}^{-}\right)$at day 10 post-DSS administration. (C) Representative contour plots and (D) mean frequency and absolute number of colonic neutrophils (CD11b+Ly6C-Ly6G+) at day 10 post-DSS administration. (E) Mean TNF- $\alpha$, IL-1 $\beta$, IL-6 production from colon tissue explants at day 10 post-DSS administration. (F) Representative H\&E staining of paraffin-embedded cross colon sections at day 10 post-DSS administration (scale bars are $100 \mu \mathrm{m}$ ). Asterisk (*) indicates area of cellular infiltration; Number sign (\#) indicates area of distorted crypt architecture; Black arrow indicates area of bleeding. Data were analyzed using a two-tailed unpaired parametric $\mathrm{t}$ test $\left(* \mathrm{p}<0.05, * * \mathrm{p}<0.01,{ }^{* * *} \mathrm{p}<0.001\right)$. (G-I) 16S rRNA gene sequencing of HMA mouse fecal bacteria. (G) Beta-Diversity was determined on weighted UniFrac distances and significance was assessed using PERMANOVA ( $\mathrm{p}=0.001$ ). (H-I) Mean relative abundance of Akkermansia sp. (H) and Escherichia-Shigella sp. (I) over time in HMA mice. Species were confirmed to be differentially abundant using ANCOM. Error bars, SE. (B, D, E) Error bars, SD. Data shown from one experiment. Dots in B, D and E represent individual mice ( $\mathrm{n}=8$ healthy-HMA mice, $\mathrm{n}=12$ UC-HMA mice). Dots in G, H, I indicate pooled mouse fecal samples at a single time point. At each time point, mouse fecal samples in each cage were pooled from 1 or 2 mice ( $n=6$ cages per group, 1 or 2 mice per cage). 

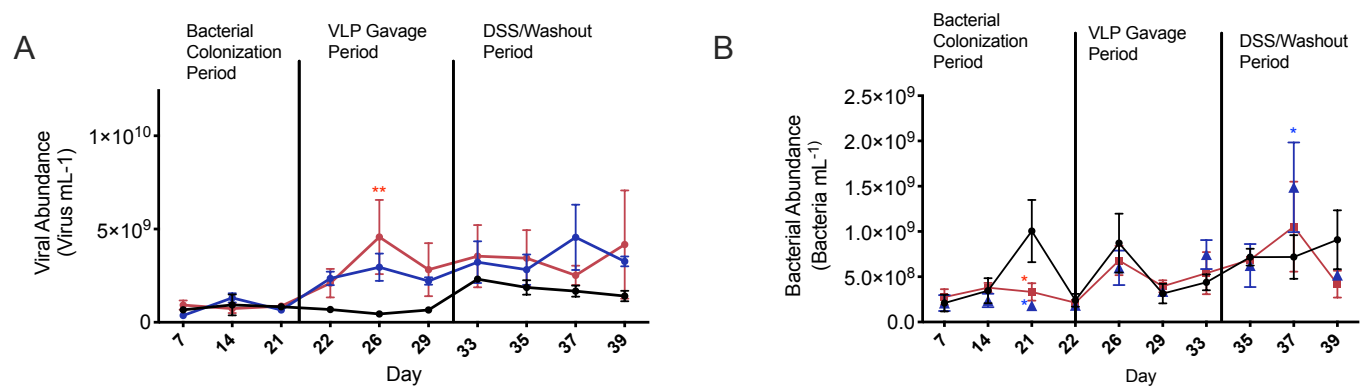

691

C

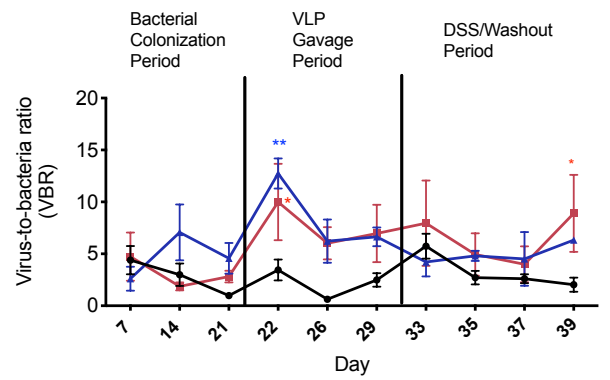

Figure 4. Healthy and UC VLP administration increases viral abundance and VBR in UC-HMA mice. (A) Viral abundance was determined from mouse fecal pellets using epifluorescence microscopy and compared to (B) bacterial abundances obtained by flow cytometry after staining with SybrGREEN I to obtain (C) VBRs. (D) Mean total viral abundance and mean total VBR post-VLP gavage were compared between treatment groups after the first dose of VLPs or PBS was given to mice. (A-C) Significance was assessed using two-way ANOVA and Dunnett's multiple comparisons test $(* p \leq 0.05, * * p \leq 0.01)$. (D) Significance was assessed using one-way ANOVA and Tukey's multiple comparison test $(* * \mathrm{p} \leq 0.01)$. Red and blue asterisks indicate significant differences between the PBS control and HMA mice given UC VLPs and healthy VLPs, respectively. Data shown is from one of two independent trials. Dots represent abundance or VBR of pooled mouse fecal samples at a single time point. At each time point, mouse fecal samples in each cage were pooled from 2 mice ( $n=3$ cages per group, 6 mice per group). Error bars, SE. UC bac, UC-HMA mice. 

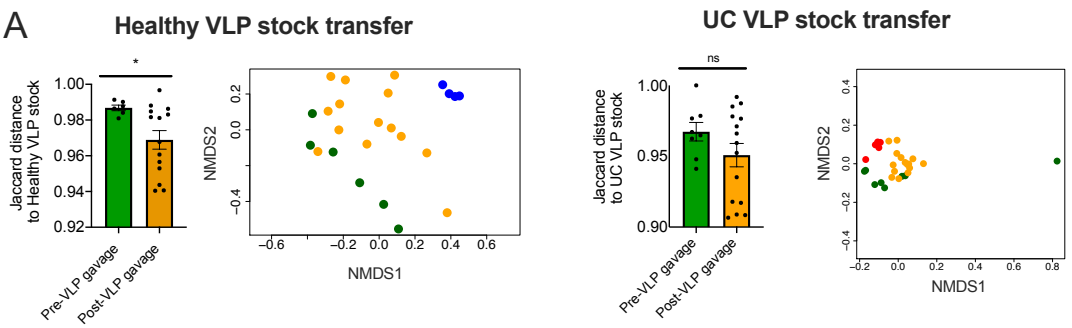

- Healthy VLP stock

- UC VLP stock

B Bacterial Colonization Period

- Pre-VLP gavage

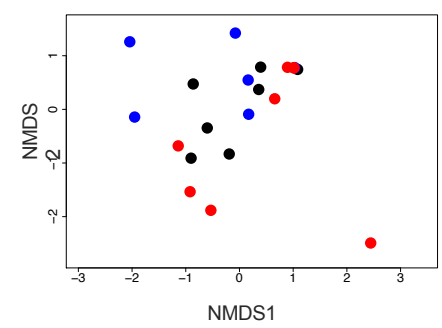

- Post-VLP gavage
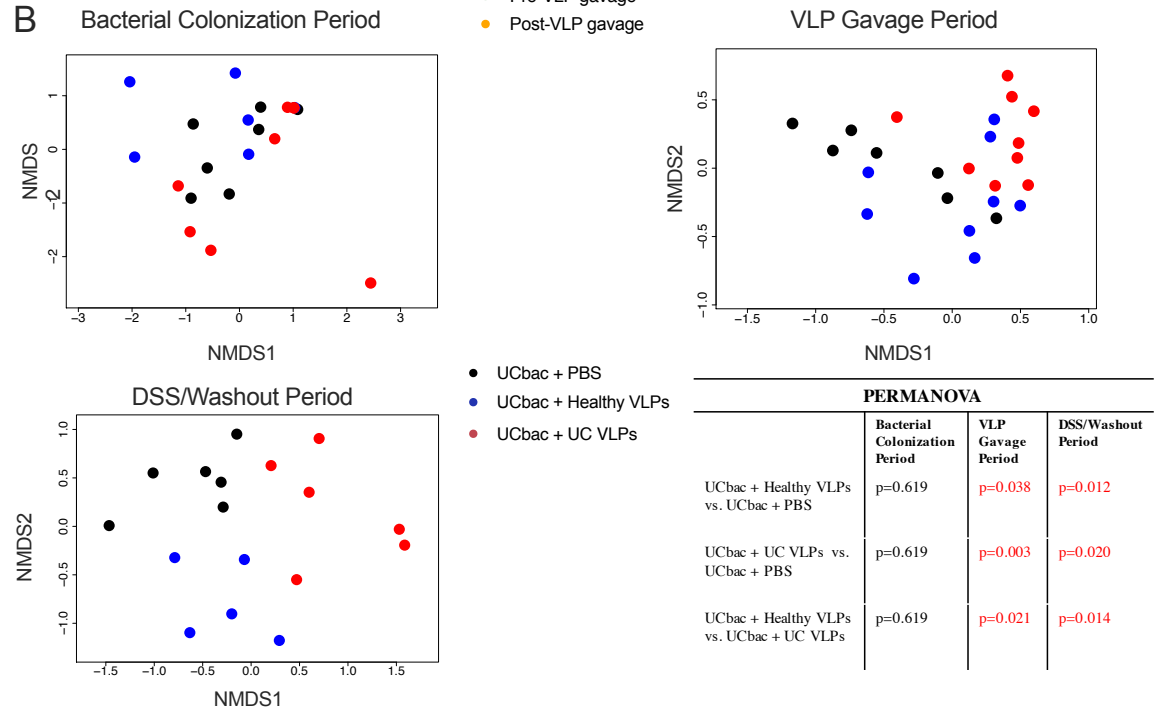

C
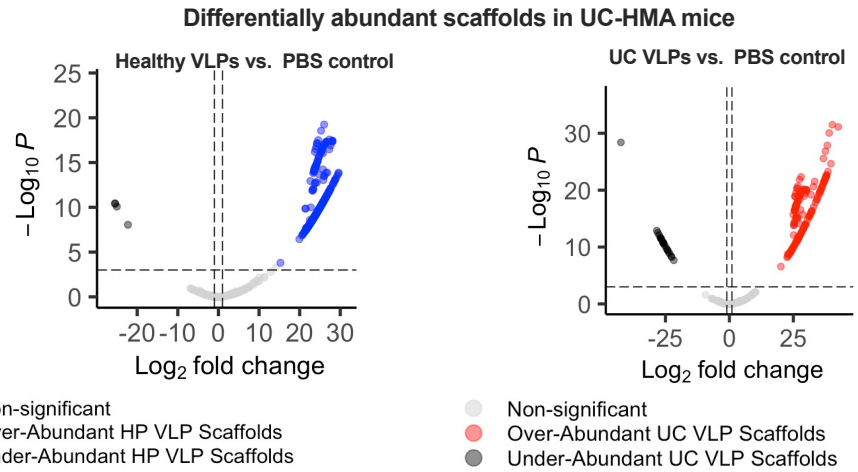

Figure 5. Differences in virome composition of UC-HMA mice after VLP gavage (A) NMDS of Jaccard distance and quantification of Jaccard distance of VCs to the healthy VLP stock (left) and UC VLP stock (right) in HMA samples before and after VLP gavage. Post-VLP gavage samples included all samples after the first dose of VLPs were added. Significant differences in Jaccard distances were assessed using an two-tailed unpaired t-test ( $\left.\mathrm{p}^{*} \leq 0.05\right)$. Error bars, SE. (B) NMDS of Bray-Curtis dissimilarity of scaffolds between HMA mice given healthy VLPs, UC VLPs or PBS during the (top-left) bacterial colonization period, (top-right) VLP gavage period or (bottom-left) DSS washout period. Significant differences in Bray-Curtis dissimilarity were assessed in each time period using adonis PERMANOVA ( $\leq 0.05$ ) (bottom-right). Dots represent pooled mouse fecal samples at a single time point. Samples from all time points of the longitudinal study were included in the NMDS and comparative analyses. (C) DESeq2 differentially abundant scaffolds between UC-HMA mice given healthy (left) and UC (right) VLPs. Dots shown in the volcano plots represent the differentially abundant scaffolds found during the VLP gavage period. Scaffolds with adjusted $\mathrm{p}$-values $<0.001$ and with $\log _{2}$ fold changes greater or less than 1 were considered differentially abundant using a two-tailed wald test ${ }^{85}$. Data shown are from the first of two independent trials represented in Fig. 1B (trial \#1). At each time point, mouse fecal samples in each cage were pooled from 2 mice ( $\mathrm{n}=3$ cages per group, 6 mice per 774 group. UCbac, UC-HMA mice). 


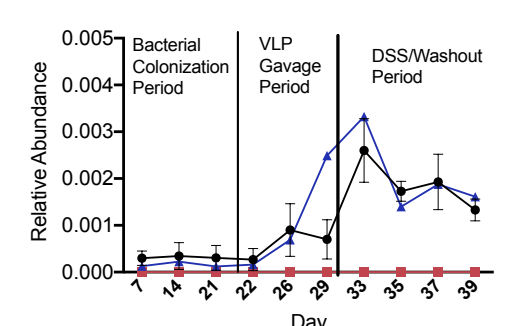

$\rightarrow U C b a c+P B S$

- UCbac + Healthy VLPs

- UCbac + UC VLPS

Enterococcus sp.

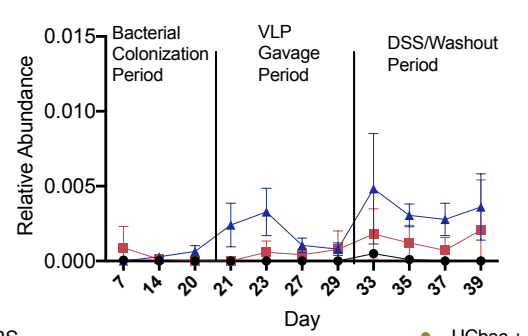

- UCbac + UC VLPs (-DSS)

- UCbac + UC VLPS (+DSS)

\pm UCbac + Heat-Killed UC VLPS (+DSS)

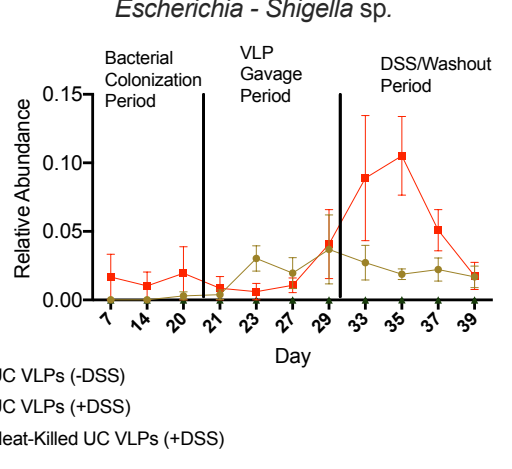

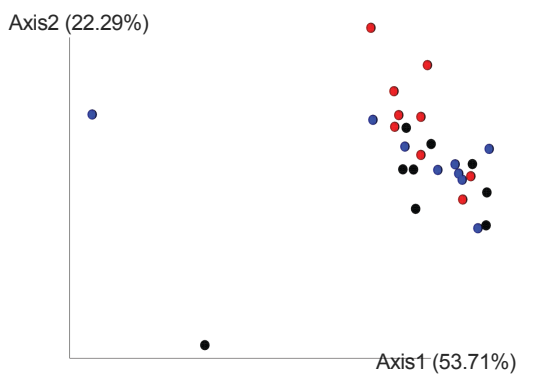

805 in UC-HMA mice (A) Mean relative abundance of Eubacterium limosum (left), Enterococcus sp. (middle) and Escherichia-Shigella sp. (right) in UC-HMA mice over time was determined using $16 \mathrm{~S}$ rRNA gene sequencing. ANCOM was used to confirm that these species were differentially abundant between treatment groups ${ }^{49}$. Error bars, SE. (B) PcoA on weighted UniFrac distance between HMA mice during the bacterial colonization period (left), VLP gavage period (middle) and DSS/washout period (right). Significant differences between weighted UniFrac distances were assessed using pairwise PERMANOVA ( $\mathrm{p} \leq 0.05$ ) (bottom). PcoA and weighted UniFrac distances shown are from the first of two independent trials represented in Fig. 1B (trial \#1). Samples from all time points were included in the PcoA and comparative analyses. Mouse fecal samples in each cage were pooled from 2 mice ( $n=3$ cages, 6 mice per treatment group). Dots represent pooled mouse fecal samples at a single time point. UCbac, UC-HMA mice. 

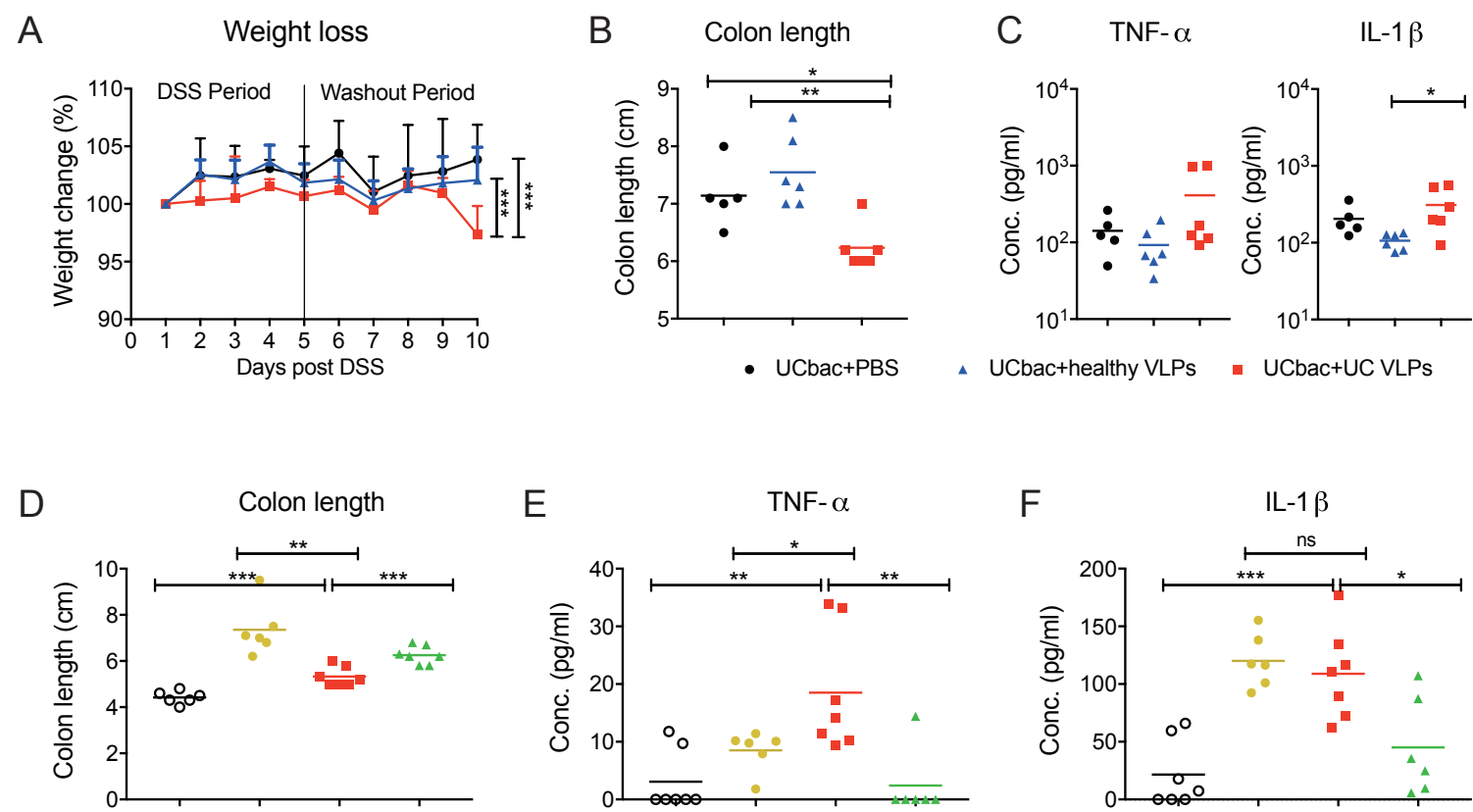

$\mathrm{E}$
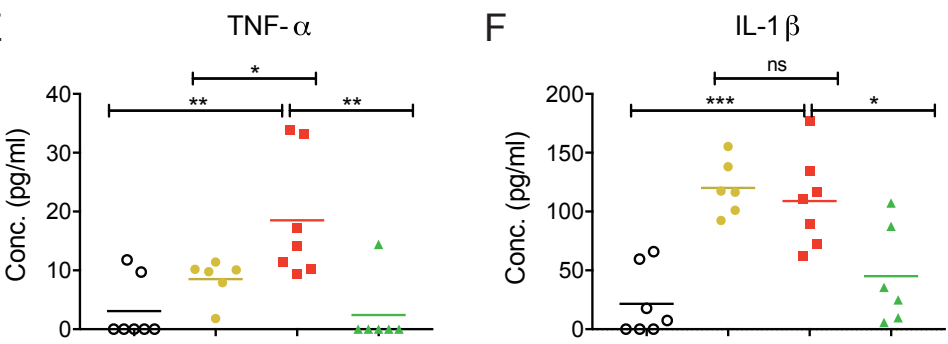

- UC VLPS (+DSS)

- UCbac+UC VLPS (-DSS) - UCbac+UC VLPS (+DSS) A UCbac+HK UC VLPS (+DSS)

822

823

824

825

826

827

828

829

830

831

832

833

834

835

836

837

838

839
Figure 7. UC VLPs exacerbate the severity of experimental colitis in the presence of gut bacteria. (A) Mean body weight change during experimental colitis induction between the indicated groups. (B) Mean colon length at day 5 post-DSS administration between the three groups. (C) Mean inflammatory cytokine production in colon tissue explant at day 5 post-DSS administration. (D) Mean length of colon at day 5 post-DSS administration of the following groups: UC bacteria colonized mice treated with UC VLPs with/without DSS challenge, UC bacteria colonized mice treated with heat-killed (HK) phages and UC VLP treatment alone. (E-F) Mean inflammatory cytokine production in colon tissue explants of the indicated groups. (A) Data were analyzed by two-way ANOVA with Bonferroni for multiple comparisons. (B-F) Data were analyzed using a two-tailed unpaired parametric $\mathrm{t}$ test $(* \mathrm{p}<0.05, * * \mathrm{p}<0.01$, $* * * \mathrm{p}<0.001)$. Error bars, SD. Data shown from one experiment. Each dot represents an individual mouse. UCbac, UC-HMA mice. 


\section{METHODS}

\section{$841 \quad$ Lead Contact}

842 Further information and requests for resources and reagents should be directed to and will be 843 fulfilled by the lead contact, Corinne Maurice (corinne.maurice@mcgill.ca).

\section{Subject details and sample collection}

846 For all experiments, fecal samples were collected from 3 UC patients (mean age: $38.66 \pm 12.97$

847 SD, mean BMI: $28.89 \pm 8.14$ SD) in remission and 3 unrelated healthy controls (mean age: 42.33

$848 \pm 13.65$ SD, mean BMI: $23.40 \pm 3.91$ SD). Samples were stored at $-80{ }^{\circ} \mathrm{C}$ until processed.

849 Participants of this study were only included if they were older than 18 years and had not taken

850 antibiotics in the 3 months prior to sample collection. UC patients were excluded if they were

851 administered treatments other than immunosuppressants. Human studies were performed with

852 approval of the McGill Ethics Research Board (REB \#A04-M27-15B).

\section{Mice}

854 Six- to 12-week-old female and male germ-free (GF) C57BL/6 mice were purchased from Charles

855 River Laboratories (Wilmington, North Carolina) and maintained in flexible film isolators at 856 McGill University. All mice had unlimited access to autoclaved mouse breeder's diet and water.

857 All mouse experiments were carried out in accordance with the approved McGill University 858 animal use protocol (7977).

\section{Experimental model}

860 HMA mice were used to assess how VLP administration impacts the gut bacterial communities

861 and colitis severity. A schematic of each experiment performed, and associated timelines are 
862 summarized in Fig. 1. Bacterial communities derived from the feces of UC patients or healthy

863 controls were administered to GF mice by oral gavage (200 uL, 1-3 x 10 cells $/ \mathrm{mL}$ ). All colonized

864 HMA mice were maintained on the same sterilized diet and water as before colonization. During

865 the 3-week bacterial reconstitution period, random fecal samples were weighed and collected from

866 each individual cage every week to monitor the reconstitution. Stool DNA was extracted by using

867 the QIAGEN QIAamp DNA stool mini Kit under a sterile biosafety cabinet. Real-time PCR

868 amplification of the V6 region of the 16S rRNA gene was performed and the concentration of

869 purified PCR products were quantified by NanoDrop spectrophotometer as a standard template.

870 (forward primer: aggattagataccctggta and reverse primer: rrcacgagctgacgac). The load of bacterial

871 DNA in feces was estimated by $16 \mathrm{~S}$ rRNA gene qPCR, and the concentration was calculated

872 according to the dilution of series of template, and normalized to the stool weight. Following a

873 period of 3 weeks of bacterial reconstitution, 1 or 4 doses of VLPs derived from the feces of UC

874 patients or healthy controls were administered to the HMA mice by oral gavage at equivalent

875 concentrations to the bacterial dose given ${ }^{86}$ over a 9-10 day period. Controls consisted of the

876 administration of PBS or heat-inactivated VLPs to HMA mice following bacterial reconstitution.

877 Following VLP treatment, 2\% DSS was administered to the mice for 5 days, followed by a 5-day

878 washout period. All mice were sacrificed at the end of the 5-day DSS washout period, and mouse

879 colons were removed to determine DSS-colitis severity. Throughout the course of the experiments,

880 mouse fecal pellets were collected to determine bacterial and VLP abundance, bacterial activity

881 and damage, to monitor disease activity index, and to determine bacterial VLP community

882 composition. 


\section{Processing of human fecal samples}

885 VLPs and bacterial communities were extracted and processed from human fecal samples, as

886 described by Khan Mirzaei et al. ${ }^{80}$ with some modifications. To acquire community-level

887 information and obtain enough material for multiple mouse experiments, two grams of each frozen

888 healthy or UC fecal sample were pooled separately, thawed under anaerobic conditions, 889 resuspended with reduced PBS (rPBS), containing 1 ug.ml ${ }^{-1}$ resazurin sodium salt and $1 \mathrm{mg} \cdot \mathrm{ml}^{-1}$

890 L-Cysteine; final concentrations, thoroughly vortexed, and centrifuged at $800 \mathrm{~g}$ for $1 \mathrm{~min}$ at $22^{\circ} \mathrm{C}$

891 to remove large debris. Pooling samples still allows for intraspecific genomic information of

892 bacterial and viral communities, despite a loss of inter-individual differences ${ }^{87}$. The resulting

893 supernatant was centrifuged at $7,000 \mathrm{~g}$ at $22^{\circ} \mathrm{C}$ for $1 \mathrm{hr}$ to separate bacterial and VLP communities.

894 Following resuspension in rPBS, bacterial concentration was determined using flow cytometry

895 (see below) ${ }^{56}$. The VLP-containing supernatant was filtered through a $0.2 \mu \mathrm{m}$ sterile syringe filter

896 (Millex-GP, Millipore Sigma, USA) concentrated by centrifugation $\left(35,000 \mathrm{~g}\right.$, $\left.4^{\circ} \mathrm{C}\right)$ for $3 \mathrm{hr}$ and

897 resuspended in SM buffer. VLP concentration was determined using epifluorescence microscopy

898 (see below) ${ }^{22}$. DNA extractions were performed on aliquots of each pooled VLP and bacterial

899 preparation for downstream sequencing analysis (see below). As a control, one aliquot of the

900 extracted VLPs were heat-inactivated by incubating the solution at $95^{\circ} \mathrm{C}$ for 20 min, followed by

901 DNAse (Ambion DNAse I, Thermo-Fisher Scientific, USA) treatment for $2 \mathrm{hr}{ }^{22}$. Bacterial and

902 viral abundances were confirmed prior to each gavage, and two hundred microliters of bacterial

903 and VLP communities were administered to mice by oral gavage at equal concentrations (1-3 x

$90410^{8}$ VLPs or bacterial cells $/ \mathrm{mL}$ ).

905

906 


\section{Induction of DSS colitis and disease activity index (DAI) evaluation}

908 HMA and GF mice received 2\% (w/v) DSS (MP Biomedicals) in drinking water for 5 days prior

909 to regular drinking water for another 5 days. The amount of DSS intake per mouse was recorded.

910 Each individual mouse was weighed to determine percentage weight changes. Fecal samples were

911 taken from each cage to clinically monitor for rectal bleeding and diarrhea. Hemoccult SENSA kit

912 (Beckman Coulter) was used to assess rectal bleeding as per the manufacturer's instructions. All

913 parameters were evaluated every 3 days.

\section{Histological evaluation of colitis}

915 HMA mice were anesthetized and sacrificed five days after completing DSS administration. 0.5

$916 \mathrm{~cm}$ of the distal colon was excised, rinsed with saline solution, fixed in $10 \%$ formalin and

917 embedded in paraffin. Sections of $4 \mu \mathrm{m}$ were stained with H\&E by the Goodman Cancer Research

918 Centre Histology Facility and assessed for histological changes in a blinded manner.

\section{Pro-inflammatory cytokine ELISA}

920 The top $0.5 \mathrm{~cm}$ of the colon was harvested and cut longitudinally, washed in PBS and excess liquid

921 was removed. The tissue was then weighed, placed in $400 \mu \mathrm{L}$ of culture medium RPMI1640

922 supplemented with $10 \%$ fetal bovine serum and culture in $37^{\circ} \mathrm{C}$ incubator overnight. The

923 supernatant was collected and transferred into a $1.5 \mathrm{~mL}$ tube and centrifuged for $5 \mathrm{~min}$ at 13,000

$924 \mathrm{rpm}$ for $4{ }^{\circ} \mathrm{C}$. The supernatant was collected, stored at $-20^{\circ} \mathrm{C}$, and used for ELISA. The mouse

925 IL-6, IL-1 $\beta$ and TNF- $\alpha$ ELISA Kits from Invitrogen (ThermoFisher) were used as per the 926 manufacturer's instructions. 


\section{Colon cell extraction}

928 The middle $5 \mathrm{~cm}$ of the colon was used to generate single cell suspensions of lamina propria cells.

929 The tissue was cut longitudinally and washed in cold Hank's Balanced Salt Solution (HBSS) +

930 EDTA buffer. The tissue was then cut into $0.5-1 \mathrm{~cm}$ sections, placed in HBSS + EDTA buffer and

931 incubated at $37^{\circ} \mathrm{C}$ for two 20 min periods with washing between incubations. The tissue was then

932 washed twice with cold HBSS buffer and digested in 5mL of digestion buffer (RPMI 1640

933 supplemented with $10 \% \mathrm{FBS}, 200 \mathrm{U} / \mathrm{mL}$ of Collagenase VIII) for $25 \mathrm{~min}$ at $37^{\circ} \mathrm{C}$. After digestion,

934 the tissue was passed through a $100 \mu \mathrm{m}$ filter and resuspended in PBS $+5 \%$ FBS for counting

935 using Trypan blue and flow cytometry analysis.

\section{Flow Cytometry of Colon Cells}

937 Colon cell suspensions were incubated with a fixable Viability dye (eFluor 506, eBioscience) for

$93825 \mathrm{~min}$ at $4{ }^{\circ} \mathrm{C}$. Cells were then incubated with Fc block $\left(7 \mathrm{~min}\right.$ at $4{ }^{\circ} \mathrm{C}$ ), followed by staining (for

93930 min at $4{ }^{\circ} \mathrm{C}$ ) with the following antibodies in appropriate combinations of fluorophores. From

940 Invitrogen: CD45.2 (104). From Biolegend: CD11b (M1/70), Ly6c (HK1.4), Ly6g (1A8). Data

941 were acquired with a FACS Canto II or LSR Fortessa (BD Biosciences) and analyzed using FlowJo

942 software (TreeStar).

\section{Processing mouse fecal samples for microbiota analyses}

944 Mouse fecal samples were processed similar to human fecal samples, with some modifications.

945 On each sampling date, fecal pellets were collected from each mouse and samples belonging to

946 the same cage were pooled in order to maximize the amount of biological material obtained. Pooled

947 pellets were resuspended in rPBS under anaerobic conditions, thoroughly vortexed and centrifuged

948 at $800 \mathrm{~g}$ for $1 \mathrm{~min}$ at $22^{\circ} \mathrm{C}$ to remove large debris. The resulting supernatant was centrifuged at 
$9496,000 \mathrm{~g}$ for $5 \mathrm{~min}$ at $22^{\circ} \mathrm{C}$ to separate VLP and bacterial communities. VLP-containing supernatant

950 was filtered through a 0.2 um sterile syringe filter (Millex-GP, Millipore Sigma, USA) to remove

951 the remaining bacteria, and phage abundance was determined using epifluorescence microscopy

952 (see below). The bacterial pellet was washed twice and resuspended in rPBS under anaerobic

953 conditions. The concentration and proportion of active and damaged bacterial cells were

954 determined using flow cytometry (see below). For the remaining fecal pellets from each cage,

955 bacterial and VLP communities were separated, as described above. Following separation,

956 bacterial and VLP DNA were extracted separately.

957 Bacterial DNA extraction and 16S rRNA gene sequencing analyses

958 Bacterial DNA was extracted from human and mouse feces-derived bacterial supernatant using

959 the DNeasy powersoil kit (Qiagen, USA) according to the manufacturer's instructions. Data

960 included from healthy and UC bacterial stocks are from four and five technical sequencing

961 replicates, respectively. The V4 region of the $16 \mathrm{~S}$ rRNA gene was amplified using the 515F/806R

962 primers, and pooled amplicons were sequenced on an Illumina Miseq with 250 bp paired-end

963 technology at the Genome Québec Centre d'expertise et de services core facility ${ }^{88}$. 16S rRNA

964 gene sequencing analysis was performed using the QIIME2 platform (v 2020.2, v2020.6) ${ }^{89}$. Read-

965 trimming, removal of chimeric reads, merging of paired-end reads, and inference of amplicon

966 sequence variants was performed using DADA2 ${ }^{90}$. In each sequencing run, read depth was rarefied

967 to the lowest number of reads that a sample contained in that run. Bacterial diversity between

968 treatment groups was assessed using Weighted UniFrac and PcoA. Taxonomic identification was

969 performed using the QIIME2 feature classifier, training a Naives-Bayes Classifier on the Silva 138

970 database ${ }^{91}$. The statistical framework, ANCOM, was used to identify differentially abundant 
971 bacterial taxa between treatment groups ${ }^{49}$. In order to disentangle the effects of VLP treatment

972 and possible isolator-specific colonization biases, bacterial species that were found to be

973 differentially abundant during the bacterial colonization period were removed from Supplementary

974 Tables. S5-8. In addition, each differentially abundant bacterial species was assigned a ranking

975 (1-3) based on likelihood that differences in relative abundance were due to VLP treatment or

976 isolator effect (1: likely due to phage treatment, 2: possibly due to phage treatment, 3: likely due

977 to isolator effect). Species classified as "likely due to phage treatment (1)" included species, where

978 reads were detected in a majority of cages in all treatment groups. Species classified as "possibly

979 due to VLP treatment" (2) included species that were found to be differentially abundant in the

980 VLP gavage and/or DSS washout period and where reads were not detected in a majority of cages

981 in all treatment groups during the bacterial colonization period. Any differentially abundant

982 species that did not meet the above criteria were classified as "likely due to isolator effect" (3).

983

984 VLP DNA extraction, amplification and sequencing

985 VLP enrichment and subsequent DNA extraction was performed as described by Reyes et al. ${ }^{22}$

986 with some modifications. Briefly, feces-derived VLP supernatant (prepared as described above)

987 was incubated with lysozyme $\left(45 \mathrm{~min}\right.$ at $\left.37^{\circ} \mathrm{C}, 50 \mathrm{mg} \cdot \mathrm{mL}^{-1}\right)$, Ambion ${ }^{\mathrm{TM}}$ Dnase $\mathrm{I}\left(1 \mathrm{hr}\right.$ at $37^{\circ} \mathrm{C}$,

$9882 \mathrm{U})$, and proteinase $\mathrm{K}\left(1 \mathrm{hr}\right.$ at $\left.37^{\circ} \mathrm{C}, 20 \mathrm{mg} \cdot \mathrm{mL}^{-1}\right)$. After addition of $5 \mathrm{M} \mathrm{NaCl}$ and $10 \%$

989 Cetyltrimethylammonium Bromide (CTAB)/0.7M NaCl solutions, samples were transferred to

990 phase lock gel tubes (QuantaBio, USA) with an equal amount of phenol:chloroform:isoamyl

991 alcohol $(25: 24: 1 \mathrm{v} / \mathrm{v}, \mathrm{pH}=8.0$, ThermoFisher Scientific, USA). The upper aqueous, DNA-

992 containing, layer was transferred to a new tube and left to precipitate overnight at $-80{ }^{\circ} \mathrm{C}$ in $100 \%$

993 ice-cold ethanol. Precipitated samples were then purified with the Zymo DNA Clean \& 
994 Concentrator 25 kit (Zymo Research, USA). Following purification, 1uL of DNA was amplified

995 in triplicate using MDA, using the GenomiPhi V3 DNA amplification kit (Cytiva, USA).

996 Amplified products were pooled, and DNA concentrations were quantified with the Qubit dsDNA

997 high-sensitivity (HS) assay kit (ThermoFisher Scientific, USA). In total, 27/90 samples from HMA

998 mice in the experiment referenced in Fig. 1B (trial \#1) were excluded from sequencing due to

999 insufficient DNA yield, including all 18 samples (2 sample collection dates) during the DSS-colitis

1000 period. For virome analyses of human stocks, 4 (healthy) and 5 (UC) technical replicates were

1001 sequenced from the same pooled fecal samples. Paired-end Illumina shotgun sequencing libraries

1002 were prepared from purified VLP-derived DNA at the Genome Québec Centre d'expertise et de

1003 services core facility. Barcoded libraries which passed quality control were pooled and sequenced

1004 on an Illumina MiSeq with 250 bp paired-end sequencing technology.

1005

1006 VLP Abundances

1007 VLPs were enumerated using epifluorescence microscopy ${ }^{33}$. Briefly, human or mouse-derived

1008 VLP supernatant were diluted in TE buffer and fixed with $1 \%$ formaldehyde for 15 min. Fixed

1009 VLPs were filtered onto $0.02 \mu \mathrm{m}$ filter membranes in triplicate (Anodisc, GE Healthcare, USA)

1010 and were stained with SYBR-Gold (2.5X concentration). On average, 25 fields of view of the

1011 stained filters were visualized and counted in triplicate using an epifluorescence microscope

1012 (Zeiss, Axioskop).

1013

\section{Bacterial Abundance and Physiology}

1015 The concentration of bacterial cells and the proportion of active and damaged bacterial cells in

1016 human and mouse feces was determined using flow cytometry, as described previously ${ }^{56}$. Briefly, 
1017 feces-derived bacterial supernatant was diluted in rPBS anaerobically, stained with SYBRGreen I

1018 (1x concentration, $15 \mathrm{~min}$ ) or PI (1x concentration, $10 \mathrm{~min}$ ) and counted using flow cytometry on

1019 a FACSCanto II (BD, USA) equipped with a 488nm laser (20 mW) and 530/30 and 585/42

1020 detection filters. Five-to-ten microliters of green fluorescent reference beads of 3.0-3.4 $\mu \mathrm{m}$

1021 (Rainbow beads, BD Biosciences) were added to each flow cytometry tube prior to acquisition to

1022 calculate bacterial concentration. The concentration of the Rainbow beads was determined by

1023 calibration with Trucount tubes (BD, USA) for each sampling date ${ }^{92}$. Total bacterial abundance

1024 was determined by SYBRGreen I staining. As previously described, ${ }^{92}$ relative nucleic acid content

1025 was used as a proxy for bacterial activity: active cells, containing more nucleic acid, were

1026 identified by their higher levels of green fluorescence. Bacterial membrane damage was assessed

1027 by PI staining: cells with high red fluorescence have lost their membrane integrity. All stainings

1028 were done in triplicates. Gating was performed using the FlowJo analysis software (v10.6.1).

1029 Gating strategy is shown in Supplementary Fig. S5A-B.

1030

1031 Prophage induction assay

1032 In order to determine whether DSS could directly cause prophage induction of fecal bacteria, we

1033 performed an in vitro induction assay ${ }^{62}$. Briefly, pooled UC fecal bacteria were grown

1034 anaerobically in triplicate at $37^{\circ} \mathrm{C}$, mixing every $15 \mathrm{~min}$, in reduced Brain Heart Infusion broth

1035 (BBL BD, Mississauga, ON, Canada) supplemented with hemin (5 $\mu \mathrm{g} / \mathrm{mL})$ and vitamin $\mathrm{K}(1$

$1036 \mu \mathrm{g} / \mathrm{mL})$. At early exponential phase, DSS was added to the growth media at final concentrations

1037 of $0 \%\left(\mathrm{H}_{2} \mathrm{O}\right.$ added $), 1 \%, 2 \%$, or $5 \%$. Bacterial growth was monitored every $15 \mathrm{~min}$ for $14 \mathrm{hr}$,

1038 measuring $\mathrm{OD}_{600}$ (Epoch 2 microplate spectrophotometer, Biotek Instruments, Winooski, VT,

1039 USA) until stationary phase was reached. Bacteria were pelleted $\left(2,000 \mathrm{xg}, 15 \mathrm{~min}, 22^{\circ} \mathrm{C}\right)$ and 
1040 VLP-containing supernatant was fixed with $1 \%$ formaldehyde and enumerated using

1041 epifluorescence microscopy. Induction was identified through a concomitant significant decrease

1042 in bacterial abundance and increase in VLPs.

1044 Bioinformatic analysis of VLP sequence data

1045 Virome analysis was performed on shotgun-sequenced VLP DNA, as described in Supplementary

1046 Fig. S1A. Briefly, raw reads (mean reads mouse samples: 176,955 \pm 21,451 SD; mean reads human

1047 VLP stocks: $1,176,926 \pm 79038$ SD) were first trimmed using Trimmomatic (v0.38) ${ }^{93}$. De novo

1048 assembly of sequencing reads was carried out with SPAdes ${ }^{94}$ (v3.12.0), using default settings.

1049 Scaffolds from each sample's assembly were then pooled and those less than $3 \mathrm{~kb}$ in length were

1050 removed ${ }^{30}$. These scaffolds were used as input for VirSorter v1.0.6 ${ }^{95}$ with the following settings:

1051 virome decontamination, DIAMOND, viromes + Gut Virome Database v1.7.2018 ${ }^{28}$. VirSorter-

1052 positive scaffolds were then used as input for VIBRANT (v1.2.1) ${ }^{29}$ with the virome mode enabled.

1053 Scaffolds which were classified as viral by VirSorter and then subsequently by VIBRANT were

1054 retained for downstream taxonomic and diversity analysis. Scaffolds classified as viral from mouse

1055 samples and scaffolds classified as viral from the pooled human stock samples were pooled, and

1056 scaffolds with $90 \%$ nucleotide identity over $90 \%$ of the shorter scaffold's length were removed

1057 using CD-HIT-EST v4.8.1 96 to form the non-redundant scaffolds library. vConTACT2 v0.9.10

1058 was used to form viral clusters from viral scaffolds ${ }^{44}$. These clusters were added to the scaffold

1059 metadata used for downstream analysis. Demovir (https://github.com/feargalr/Demovir) was used

1060 to assign taxonomic identities to the viral scaffolds. This tool uses a voting approach for taxonomy

1061 based on protein homology to a viral subset of the TrEMBL database from UniProt. Additionally,

1062 scaffolds were identified as being a crAss-like phage if they had BLASTn alignments to a 
1063 crAssphage database with an e-value $\leq 10^{-10}$, covering $\geq 90 \%$ of the scaffold's length, and over

$106450 \%$ nucleotide identity ${ }^{30,97}$. We used CrisprOpenDB, which uses BLASTn alignment to a

1065 CRISPR spacer database to assign bacterial genus predictions for each viral scaffold ${ }^{48}$. Default

1066 settings were used, allowing up to 2 mismatches between a scaffold and spacer. Using VIBRANT,

1067 scaffolds were identified as temperate based on the presence of the integrase gene ${ }^{29}$. We used

1068 Bowtie2 v2.3.5 ${ }^{98}$ to map trimmed reads back to assembled viral scaffolds. SAMtools v1.9 ${ }^{99}$ was

1069 used to convert SAM to BAM files. Custom Python scripts were used to create scaffold metadata

1070 files and abundance matrices. These scripts are available at the GitHub

1071 (https://github.com/MauriceLab/phage_colitis). Scaffolds were only included in analyses for a

1072 given sample if coverage was $\geq 1 \mathrm{x}$ over $\geq 75 \%$ of the scaffold length ${ }^{100}$. In order to determine

1073 differentially abundant viral scaffolds, raw counts matrices were input into DESeq2 v.1.3.0 ${ }^{57}$. The

1074 standard DESeq2 workflow was performed, using "poscounts" for estimation of size factors. In

1075 each comparison, scaffolds with adjusted p-values $<0.001$ and with $\log _{2}$ fold changes greater or

1076 less than 1 were considered differentially abundant ${ }^{85}$. Bray-Curtis dissimilarity and Jaccard

1077 distances for diversity analyses were performed on matrices normalized by total coverage using

1078 Vegan v2.5-7. Visualization of NMDS and relative abundance plots was performed using the

1079 ggplot2 v3.3.3 package.

1080

\section{Quantification and Statistical Analysis}

1082 Data were analyzed using GraphPad Prism software (v 8.4.3). Specific tests for determining

1083 statistical significance are indicated in the figure legends. $\mathrm{P}$ values $<0.05$ were considered

1084 statistically significant, except for DESeq2 virome analyses, where $\mathrm{p}$ values $<0.001$ and values

1085 with $\log _{2}$ fold change greater or less than 1 were considered statistically significant. Differences in 
1086 viral diversity were assessed using NMDS of Bray-Curtis dissimilarity and Jaccard distance in R

1087 v.4.0.5 (R Core Team, 2021) using the Vegan v2.5-7 and ggplot2 v3.3.3 packages. Adonis

1088 PERMANOVA was used to determine significant differences in Bray-Curtis Dissimilarity

1089 between the viromes of HMA mice given healthy VLPs, UC VLPs or PBS, using the Vegan v2.5-

10907 package. Differentially abundant bacterial species were identified using ANCOM. PcoA on

1091 weighted UniFrac distances were used to determine differences in bacterial diversity between

1092 HMA mice. Significant differences on weighted UniFrac distance between HMA mice was

1093 determined using PERMANOVA, using the QIIME2 platform (v2020.2, v2020.6) ${ }^{89}$. P-values for

1094 all PERMANOVA tests were adjusted for multiple comparisons using Benjamini-Hochberg.

1095

1096 DECLARATIONS

\section{Ethics approval and consent to participate}

1098

1099 Human studies were performed with approval of the McGill Ethics Research Board (REB \#A04-

1100 M27-15B). All participants provided informed consent prior to donating samples. All mouse

1101 experiments were carried out in accordance with the approved McGill University animal use

1102 protocol (7977).

\section{Availability of data and materials}

1106 Custom scripts and code used for data analysis are available at

1107 https://github.com/MauriceLab/phage_colitis. Sequencing reads for bacterial 16S rRNA gene 1108 sequencing and VLP shotgun metagenomics are available on NCBI SRA. Bacterial 16S rRNA 1109 gene sequencing reads are available using accession number: PRJNA734661. VLP shotgun 
1110 metagenomic reads are available using accession number: PRJNA732769. This study did not

1111 generate new unique reagents.

1112 Competing interests

1113 The authors declare no competing interests.

1114 Funding

1115 This work was funded by the Kenneth Rainin Foundation (Innovator Award 2016-1280).

\section{Authors' Contributions}

1117 A.S., Y.L. and M.K.M. designed and performed experiments, analyzed results and wrote the

1118 manuscript. A.S. and M.S. performed bioinformatic analyses. R.S. provided critical animal

1119 resources. C.F.M. and I.L.K. conceived the project, designed experiments and wrote the 1120 manuscript.

\section{Acknowledgements}

1122 A special thanks to the Comparative Medicine and Animal Resources Centre of McGill University,

1123 especially Anna Jimenez for her technical support using germ-free mice. We also appreciate the

1124 support of Genome Quebec for microbial sequencing services. The flow cytometry work was

1125 performed in the Flow Cytometry Core Facility of the Life Science Complex and supported by

1126 funding from the Canadian Foundation for Innovation. AS is supported by a Canadian Institute of

1127 Health Research (CIHR) fellowship (170921). YL was supported by a Richard and Edith Strauss

1128 Postdoctoral Fellowship in Medicine. ILK is a Canada Research Chair in Barrier Immunity. CFM 
1129 is a Canada Research Chair in Gut Microbial Physiology and CIFAR Azrieli Global Scholar,

1130 Humans \& Microbiome Program.

\section{ADDITIONAL FILES}

\section{Additional File 1:}

1133 Supplementary Figure S1. Virome analyses on stock and mouse VLPs. Related to Figure 2 and

1134 Figure 5. Supplementary Figure S2. PcoA on Weighted UniFrac distances in HMA mice given

1135 VLPs. Related to Figure 3 and Figure 6. Supplementary Figure S3. Experimental colitis severity 1136 of UC-HMA and healthy-HMA mice given a single dose of healthy or UC VLPs. Related to Figure

1137 3. Supplementary Figure S4. Viral and bacterial abundance in UC-HMA mice. Related to Figure

1138 4. Supplementary Figure S5. Bacterial physiology of HMA mice. Related to Figure 4.

1139 Supplementary Figure S6. Human microbiota protects mice from experimental colitis. Related

1140 to Figure 7. Supplementary Table. S2. PERMANOVA on weighted UniFrac distances between

1141 UC or healthy-HMA mice given healthy VLPs or UC VLPs. Related to Figure 3 and Figure 6.

1142 Supplementary Table. S5. Differentially abundant species during VLP gavage period in HMA

1143 mice given healthy VLPs, UC VLPs, or PBS. Related to Figure 6. Supplementary Table. S6.

1144 Differentially abundant species during DSS/washout period in HMA mice given healthy VLPs,

1145 UC VLPs, or PBS. Related to Figure 6. Supplementary Table. S7. Differentially abundant

1146 species during VLP gavage period in HMA mice given UC VLPs (+/- DSS), or heat-killed UC

1147 VLPs. Related to Figure 6. Supplementary Table. S8. Differentially abundant species during

1148 DSS/ washout period in HMA mice given UC VLPs (+/- DSS) or heat-killed UC VLPs. Related

1149 to Figure 6. 


\section{Additional File 2:}

1153 Table.S1. VLP scaffolds and bacterial phyla and genera in pooled healthy and UC stocks. Related

1154 to Figure 2. Healthy VLP stock scaffolds and annotations (tab 1), UC VLP stock scaffolds and 1155 annotations ( $\operatorname{tab} 2$ ), bacterial phyla relative abundance (tab 3), bacterial genera relative abundance $1156(\operatorname{tab} 4)$.

\section{Additional File 3:}

1158 Table. S3. VLP scaffolds shared between stocks and HMA mice post-VLP gavage. Related to

1159 Figure 5. VLP scaffolds shared between healthy VLP stock and UC-HMA mice post-VLP gavage 1160 (tab 1), VLP scaffolds shared between the UC VLP stock and UC-HMA mice post-VLP gavage 1161 (tab 2), scaffold metadata (tab 3).

\section{Additional File 4:}

1163 Table. S4. Differentially abundant viral scaffolds. Related to Figure 5. Healthy VLPs vs. PBS 1164 control, phage gavage period (tab 1). UC VLPs vs. PBS control, phage gavage period (tab 2). 1165 Healthy VLPs vs. PBS control, DSS washout period (tab 3). UC VLPs vs. PBS control, DSS 1166 washout period (tab 4). Post-DSS vs. pre-DSS, PBS control (tab 5). Post-DSS vs. pre-DSS, HMA1167 mice treated with healthy VLPs (tab 6). Post-DSS vs. pre-DSS, HMA mice treated with UC VLPs $1168(\operatorname{tab} 7)$. 


\section{REFERENCES}

11781 Valdes, A. M., Walter, J., Segal, E. \& Spector, T. D. Role of the gut microbiota in nutrition and health. Bmj 361, k2179, doi:10.1136/bmj.k2179 (2018).

11802 Belkaid, Y.\& Hand, T. W. Role of the microbiota in immunity and inflammation. Cell $1181 \quad$ 157, 121-141, doi:10.1016/j.cell.2014.03.011 (2014).

11823 Arpaia, N. et al. Metabolites produced by commensal bacteria promote peripheral 1183 regulatory T-cell generation. Nature 504, 451-455, doi:10.1038/nature12726 (2013).

11844 Atarashi, K. et al. Treg induction by a rationally selected mixture of Clostridia strains 1185 from the human microbiota. Nature 500, 232-236, doi:10.1038/nature12331 (2013).

11865 Furusawa, Y. et al. Commensal microbe-derived butyrate induces the differentiation of 1187 colonic regulatory T cells. Nature 504, 446-450, doi:10.1038/nature12721 (2013).

11886 Pabst, O. et al. Adaptation of solitary intestinal lymphoid tissue in response to microbiota and chemokine receptor CCR7 signaling. J Immunol 177, 6824-6832, doi:doi:10.4049/jimmunol.177.10.6824 (2006).

Lloyd-Price, J. et al. Multi-omics of the gut microbial ecosystem in inflammatory bowel diseases. Nature 569, 655-662, doi:https://doi.org/10.1038/s41586-019-1237-9 (2019).

11938 Nemoto, H. et al. Reduced diversity and imbalance of fecal microbiota in patients with 1194 ulcerative colitis. Dig Dis Sci 57, 2955-2964, doi:10.1007/s10620-012-2236-y (2012).

11959 Parada Venegas, D. et al. Short Chain Fatty Acids (SCFAs)-Mediated Gut Epithelial and Immune Regulation and Its Relevance for Inflammatory Bowel Diseases. Frontiers of Immunology 10, 277, doi:10.3389/fimmu.2019.00277 (2019).

119810 Rigottier-Gois, L. Dysbiosis in inflammatory bowel diseases: the oxygen hypothesis. 1199 ISME 7, 1256-1261, doi:10.1038/ismej.2013.80 (2013).

120011 Bel, S. et al. Reprogrammed and transmissible intestinal microbiota confer diminished susceptibility to induced colitis in TMF -/- mice. PNAS 13, 4964-4969, doi:doi: 10.1073/pnas.1319114111 (2014).

120312 Bian, X. et al. Administration of Akkermansia muciniphila Ameliorates Dextran Sulfate Sodium-Induced Ulcerative Colitis in Mice. Frontiers in Microbiology 10, doi:10.3389/fmicb.2019.02259 (2019).

120613 Garrett, W. S. et al. Enterobacteriaceae act in concert with the gut microbiota to induce 
120914 Lee, J. Y. et al. High-Fat Diet and Antibiotics Cooperatively Impair Mitochondrial

1210 Bioenergetics to Trigger Dysbiosis that Exacerbates Pre-inflammatory Bowel Disease. Cell Host Microbe 28, 273-284.e276, doi:10.1016/j.chom.2020.06.001 (2020).

121215 Martín, R. et al. Faecalibacterium prausnitzii prevents physiological damages in a chronic low-grade inflammation murine model. BMC Microbiology 15, 67, doi:doi:10.1186/s12866-015-0400-1 (2015).

121516 Graham, D. B. \& Xavier, R. J. Pathway paradigms revealed from the genetics of inflammatory bowel disease. Nature 578, 527-539, doi:10.1038/s41586-020-2025-2 (2020).

121817 Jostins, L. et al. Host-microbe interactions have shaped the genetic architecture of inflammatory bowel disease. Nature 491, 119-124, doi:10.1038/nature11582 (2012). marine microbial realm. Nature Microbiology 3, 754-766, doi:doi: 10.1038/s41564-0180166-y (2018). stranded DNA viruses in human feces. Appl Environ Microbiol 77, 8062-8070, doi:10.1128/aem.06331-11 (2011).

21 Rasmussen, T. S. et al. Faecal virome transplantation decreases symptoms of type 2 diabetes and obesity in a murine model. Gut 69, 2122-2130, doi:10.1136/gutjnl-2019320005 (2020).

22 Reyes, A., Wu, M., McNulty, N. P., Rohwer, F. L. \& Gordon, J. I. Gnotobiotic mouse model of phage-bacterial host dynamics in the human gut. Proc Natl Acad Sci U S A 110, 20236-20241, doi:10.1073/pnas.1319470110 (2013).

Draper, L. A. et al. Autochthonous faecal viral transfer (FVT) impacts the murine microbiome after antibiotic perturbation. BMC Biology 18, 1-14 (2020). 348, doi:10.3389/fcimb.2019.00348 (2019). 
$124326 \quad$ Khan Mirzaei, M. \& Maurice, C. F. Ménage à trois in the human gut: interactions between host, bacteria and phages. Nat Rev Microbiol 15, 397-408, doi:10.1038/nrmicro.2017.30 (2017).

27 Camarillo-Guerrero, L. F., Almeida, A., Rangel-Pineros, G., Finn, R. D. \& Lawley, T. D. Massive expansion of human gut bacteriophage diversity. Cell 184, 1098-1109.e1099, doi:10.1016/j.cell.2021.01.029 (2021). Virome Diversity in the Human Gut. Cell Host Microbe 28, 724-740.e728, doi:10.1016/j.chom.2020.08.003 (2020).

29 Kieft, K., Zhou, Z. \& Anantharaman, K. VIBRANT: automated recovery, annotation and curation of microbial viruses, and evaluation of viral community function from genomic sequences. Microbiome 8 (2020).

125530 Shkoporov, A. N. et al. The Human Gut Virome Is Highly Diverse, Stable, and 1256 Individual Specific. Cell Host and Microbe 26, 527-541 (2019).

125731 Minot, S. et al. Rapid evolution of the human gut virome. Proc Natl Acad Sci U S A 110, 12450-12455, doi:10.1073/pnas.1300833110 (2013).

32 Fujimoto, K. et al. Metagenome Data on Intestinal Phage-Bacteria Associations Aids the Development of Phage Therapy against Pathobionts. Cell Host Microbe 28, 380389.e389, doi:10.1016/j.chom.2020.06.005 (2020).

33 Reyes, A. et al. Viruses in the faecal microbiota of monozygotic twins and their mothers. Nature 466, 334-338, doi:10.1038/nature09199 (2010).

126434 Zuo, T. et al. Human-Gut-DNA Virome Variations across Geography, Ethnicity, and Urbanization. Cell Host Microbe 28, 741-751.e744, doi:10.1016/j.chom.2020.08.005 (2020).

126735 Kim, M.-S. \& Bae, J.-W. Lysogeny is prevalent and widely distributed in the murine gut microbiota. ISME 12, 1127 - 1141, doi:10.1038/s41396-018-0061-9 (2018). human gut virus crAssphage. Nat Microbiol 4, 1727-1736, doi:10.1038/s41564-0190494-6 (2019). doi:10.1016/j.chom.2019.10.009 (2019). 
127839 Norman, J. M. et al. Disease-specific alterations in the enteric virome in inflammatory bowel disease. Cell 160, 447-460, doi:10.1016/j.cell.2015.01.002 (2015).

$128040 \quad$ Zuo, T. et al. Gut mucosal virome alterations in ulcerative colitis. Gut 68, doi:doi:

$1281 \quad 10.1136 /$

1282 gutjnl-2018-318131). (2019).

128341 Cornuault, J. K. et al. Phages infecting Faecalibacterium prausnitzii belong to novel viral genera that help to decipher intestinal viromes. Microbiome 6, 65, doi:10.1186/s40168-

1285 018-0452-1 (2018).

1286

Duerkop, B. A. et al. Murine colitis reveals a disease-associated bacteriophage community. Nat Microbiol 3, 1023-1031, doi:10.1038/s41564-018-0210-y (2018).

43 Okayasu, I. et al. A novel method in the induction of reliable experimental acute and chronic ulcerative colitis in mice. Gastroenterology 98, 694-702, doi:10.1016/00165085(90)90290-h (1990).

129144 Bin Jang, H. et al. Taxonomic assignment of uncultivated prokaryotic virus genomes is 0100-8 (2019).

$1294 \quad 45$ Zhou, Y.\& Zhi, F. Lower Level of Bacteroides in the Gut Microbiota Is Associated with Inflammatory Bowel Disease: A Meta-Analysis. Biomed Res Int 2016, 5828959, doi:10.1155/2016/5828959 (2016).

129746 Alam, M. T. et al. Microbial imbalance in inflammatory bowel disease patients at different taxonomic levels. Gut Pathog 12, 1, doi:10.1186/s13099-019-0341-6 (2020).

129947 Zakerska-Banaszak, O. et al. Dysbiosis of gut microbiota in Polish patients with ulcerative colitis: a pilot study. Sci Rep 11 (2021).

130148 Dion, M. B. et al. Streamlining CRISPR spacer-based bacterial host predictions to decipher the viral dark matter. Nucleic Acids Res 49, 3127-3138, doi:10.1093/nar/gkab133 (2021).

49 Mandal, S. et al. Analysis of composition of microbiomes: a novel method for studying microbial composition. Microb Ecol Health Dis 29, doi:doi: 10.3402/mehd.v26.27663 (2015).

130750 Britton, G. J. et al. Microbiotas from Humans with Inflammatory Bowel Disease Alter

$131051 \quad$ Natividad, J. M. et al. Ecobiotherapy Rich in Firmicutes Decreases Susceptibility to the Balance of Gut Th17 and ROR $\gamma \mathrm{t}(+)$ Regulatory T Cells and Exacerbate Colitis in Mice. Immunity 50, 212-224.e214, doi:10.1016/j.immuni.2018.12.015 (2019). 
131352 Png, C. W. et al. Mucolytic bacteria with increased prevalence in IBD mucosa augment

1314

1315

1316

1317

1318

1319

1320

132

1322

1323

1324

1325

1326

1327

1328

1329

1330

1331

1332

1333

133460

1335

1336

1337

1338

1339

1340

1341

1342

1343

1344

1345

1346

1347

1348 in vitro utilization of mucin by other bacteria. Am J Gastroenterol 105, 2420-2428, doi:10.1038/ajg.2010.281 (2010).

53 Abedon, S. T., Kuhl, S. J., Blasdel, B. G. \& Kutter, E. M. Phage treatment of human infections. Bacteriophage 1, 66-85, doi:10.4161/bact.1.2.15845 (2011).

54 Bull, J. J., Vegge, C. S., Schmerer, M., Chaudhry, W. N. \& Levin, B. R. Phenotypic resistance and the dynamics of bacterial escape from phage control. PLoS One 9, e94690, doi:10.1371/journal.pone.0094690 (2014).

55 Silveira, C. B. \& Rohwer, F. L. in NPJ Biofilms Microbiomes Vol. 216010 (2016).

56 Maurice, C. F., Haiser, H. J. \& Turnbaugh, P. J. Xenobiotics shape the physiology and gene expression of the active human gut microbiome. Cell 152, 39-50, doi:10.1016/j.cell.2012.10.052 (2013).

57 Love, M. I., Huber, W. \& Anders, S. Moderated estimation of fold change and dispersion for RNA-seq data with DESeq2. Genome Biology 15, doi:10.1186/s13059-014-0550-8 (2014).

58 Kanauchi, O. et al. Eubacterium limosum ameliorates experimental colitis and metabolite of microbe attenuates colonic inflammatory action with increase of mucosal integrity. World J Gastroenterol 12, 1071-1077, doi:10.3748/wjg.v12.i7.1071 (2006).

59 Takahashi, K. et al. Effect of Enterococcus faecalis 2001 on colitis and depressive-like behavior in dextran sulfate sodium-treated mice: involvement of the brain-gut axis. Journal of Neuroinflammation 16 (2019).

60 Seishima, J. et al. Gut-derived Enterococcus faecium from ulcerative colitis patients promotes colitis in a genetically susceptible mouse host. Genome Biol 20, 252, doi:10.1186/s13059-019-1879-9 (2019).

61 Frank, D. N. et al. Molecular-phylogenetic characterization of microbial community imbalances in human inflammatory bowel diseases. Proc Natl Acad Sci U S A 104, 13780-13785, doi:10.1073/pnas.0706625104 (2007).

62 Sutcliffe, S. G., Shamash, M., Hynes, A. P. \& Maurice, C. F. Common Oral Medications Lead to Prophage Induction in Bacterial Isolates from the Human Gut. Viruses 13, 455 (2021).

63 Otsuji, N., Sekiguchi, M., Iijima, T. \& Takagi, Y. Induction of phage formation in the lysogenic Escherichia coli K-12 by mitomycin C. Nature 184(Suppl 14), 1079-1080, doi:10.1038/1841079b0 (1959).

64 Jiang, S. C. \& Paul, J. H. Occurrence of lysogenic bacteria in marine microbial communities as determined by prophage induction. Mar Ecol Prog Ser 142, 27-38 (1996). 
134965 Braga, L. P. P. et al. Impact of phages on soil bacterial communities and nitrogen availability under different assembly scenarios. Microbiome 8, doi:doi: 10.1186/s40168020-00822-z (2020). of the Microbiome. Cell Host Microbe 25, 195-209, doi:10.1016/j.chom.2019.01.017 (2019).

67 Gogokhia, L.et al. Expansion of Bacteriophages Is Linked to Aggravated Intestinal Inflammation and Colitis. Cell Host Microbe 25, 285-299.e288, doi:10.1016/j.chom.2019.01.008 (2019).

Schirmer, M. et al. Dynamics of metatranscription in the inflammatory bowel disease gut microbiome. Nat Microbiol 3, 337-346, doi:10.1038/s41564-017-0089-z (2018).

$136370 \quad$ Kiesler, P., Fuss, I. J. \& W, S. Experimental Models of Inflammatory Bowel Diseases. Cellular and Molecular Gastroenterology and Hepatology 1, 154-170, doi:https://doi.org/10.1016/j.jcmgh.2015.01.006 (2015).

$136671 \quad$ Krych, Ł. et al. Have you tried spermine? A rapid and cost-effective method to eliminate dextran sodium sulfate inhibition of PCR and RT-PCR. Journal of Microbiological doi:10.1186/s12864-014-1207-4 (2015). uncultured single-stranded and double-stranded DNA viruses. Appl Environ Microbiol 77, 7663-7668, doi:10.1128/aem.00289-11 (2011). metagenomic surveys of saliva DNA viruses. Microbiome 6, doi:10.1186/s40168-0180507-3 (2018). 
$138477 \quad$ Fouladi, F. et al. Sequence variant analysis reveals poor correlations in microbial taxonomic abundance between humans and mice after gnotobiotic transfer. ISME 14, $1809-1820(2020)$.

138778 Arrieta, M. C., Walter, J. \& Finlay, B. B. Human Microbiota-Associated Mice: A Model with Challenges. Cell Host Microbe 19, 575-578, doi:10.1016/j.chom.2016.04.014 (2016).

139079 Džunková, M. et al. Defining the human gut host-phage network through single-cell viral

80 Khan Mirzaei, M. et al. Bacteriophages Isolated from Stunted Children Can Regulate Gut Bacterial Communities in an Age-Specific Manner. Cell Host Microbe 27, 199-212.e195, doi:10.1016/j.chom.2020.01.004 (2020).

81 Li, Y., Handley, S. A. \& Baldridge, M. T. The dark side of the gut: Virome-host interactions in intestinal homeostasis and disease. J Exp Med 218, doi:10.1084/jem.20201044 (2021). Escherichia coli Pathobionts Associated with Inflammatory Bowel Disease. Clin Microbiol Rev 32, doi:10.1128/cmr.00060-18 (2019).

$140183 \quad$ Machiels, K. et al. A decrease of the butyrate-producing species Roseburia hominis and Faecalibacterium prausnitzii defines dysbiosis in patients with ulcerative colitis. Gut 63, 1275-1283, doi:10.1136/gutjnl-2013-304833 (2014).

140484 Huda-Faujan, N. et al. The impact of the level of the intestinal short chain Fatty acids in inflammatory bowel disease patients versus healthy subjects. Open Biochem J 4, 53-58, doi:10.2174/1874091x01004010053 (2010).

85 Mangalea, M. R. et al. Individuals at risk for rheumatoid arthritis harbor differential intestinal bacteriophage communities with distinct metabolic potential. Cell Host Microbe 29, 726-739.e725, doi:10.1016/j.chom.2021.03.020 (2021).

$141387 \quad$ Schlötterer, C., Tobler, R., Kofler, R. \& Nolte, V. Sequencing pools of individuals -

Staley, C. et al. Stable engraftment of human microbiota into mice with a single oral gavage following antibiotic conditioning. Microbiome 5, doi:doi: 10.1186/s40168-017763 (2014). 
141989 Bolyen, E. et al. Reproducible, interactive, scalable and extensible microbiome data science using QIIME 2. Nature Biotechnology 37, 852-857 (2019).

Callahan, B. J. et al. DADA2: High-resolution sample inference from Illumina amplicon data. Nature Methods 13, 581-583, doi:10.1038/nmeth.3869 (2016).

$142391 \quad$ Bokulich, N. A. et al. Optimizing taxonomic classification of marker-gene amplicon sequences with QIIME 2's q2-feature-classifier plugin. Microbiome 6, 90, doi:10.1186/s40168-018-0470-z (2018).

92 Maurice, C. F. \& Turnbaugh, P. J. Quantifying and Identifying the Active and Damaged Subsets of Indigenous Microbial Communities Methods in Enzymology. Methods in Enzymology 531, 91-107, doi:doi: 10.1016/B978-0-12-407863-5.00005-8. (2013). sequence data. Bioinformatics 30, 2114-2120 (2014).

94 Bankevich, A. et al. SPAdes: a new genome assembly algorithm and its applications to single-cell sequencing. Journal of computational biology 19, 455-477, doi:10.1089/cmb.2012.0021 (2014). microbial genomic data. PeerJ 3, e985, doi:10.7717/peerj.985 (2015). generation sequencing data. Bioinformatics 28, 3150-3152, doi:10.1093/bioinformatics/bts565 (2012).

144398 Langmead, B. \& Salzberg, S. L. Fast gapped-read alignment with Bowtie 2. Nat Methods 9, 357-359, doi:10.1038/nmeth.1923 (2012). Abundant Virus in the Human Gut. Cell Host Microbe 24, 653-664.e656, doi:10.1016/j.chom.2018.10.002 (2018). 